\title{
On the Equations Defining Abelian Varieties. III
}

\section{Citation}

Mumford, David B. 1967. On the equations defining abelian varieties. III. Inventiones

Mathematicae 3(3): 215-244.

\section{Published Version}

doi:10.1007/BF01425401

\section{Permanent link}

http://nrs.harvard.edu/urn-3:HUL.InstRepos:3597244

\section{Terms of Use}

This article was downloaded from Harvard University's DASH repository, and is made available under the terms and conditions applicable to Other Posted Material, as set forth at http:// nrs.harvard.edu/urn-3:HUL.InstRepos:dash.current.terms-of-use\#LAA

\section{Share Your Story}

The Harvard community has made this article openly available.

Please share how this access benefits you. Submit a story.

\section{Accessibility}




\title{
On the Equations Defining Abelian Varieties. III*
}

\author{
D. Mumford (Cambridge, Mss.)
}

\section{Contents}

§10. Non-Degenerate Theta Functions . . . . . . . . . . . . . . . 215

$\S 11$. Satake's Compactification . . . . . . . . . . . . . . . . . . . . . 228

$\S 12$. Analytic Theta Functions . . . . . . . . . . . . . . . . . . . . . . 2236

\section{$\S 10$. Non-Degenerate Theta Functions}

The third part of this paper is devoted (1) to a complete description of the boundary of the moduli space for abelian varieties described in $\S 9$, and (2) to connecting our theory with the classical theory of theta functions. We begin by defining a theta function in a coordinate-free manner and investigating how and under what non-degeneracy restrictions we can construct a tower of abelian varieties having this as its theta function. Our goal is to find an inverse to the moduli map $\Theta$ described in $\S 9$. Fix

o) an algebraically closed field $k$, char $(k) \neq 2$;

i) a $2 g$-dimensional vector space $V$ over $\boldsymbol{Q}_{2}$;

ii) a skew-symmetric bi-multiplicative map:

i.e.,

$$
e: V \times V \rightarrow\left\{2^{n} \text {-th roots of } 1 \text { in } k\right\}
$$

$$
\begin{aligned}
e(\alpha, \alpha) & =1 \\
e(\alpha \cdot \beta, \gamma) & =e(\alpha, \gamma) \cdot e(\beta, \gamma) \\
e(\alpha, \beta \cdot \gamma) & =e(\alpha, \beta) \cdot e(\alpha, \gamma)
\end{aligned}
$$

iii) a maximal isotropic lattice $\Lambda \subset V$ (i.e., a compact, open subgroup such that $e(\alpha, \beta)=1$, all $\alpha, \beta \in \Lambda$, maximal with this property);

iv) a quadratic character

such that

$$
e_{*}: \frac{1}{2} \Lambda / \Lambda \rightarrow\{ \pm 1\}
$$

all $\alpha, \beta \in \frac{1}{2} \Lambda$.

$$
e_{*}(\alpha+\beta) e_{*}(\alpha) e_{*}(\beta)=e(\alpha, \beta)^{2},
$$

$\star$ Part I of this paper has been published in Inventiones math. Vol. 1, pp. 287-354 and part II in Vol. 3, pp. 75-135. 
We assume, however, that via a suitable isomorphism $V \cong Q_{2}^{2 g}, \Lambda \cong Z_{2}^{2 g}$, and $e, e_{*}$ have the form defined in $\S 9$. In fact, this is nearly always the case: if we write

$$
e_{*}(\alpha)=(-1)^{Q(\alpha)}
$$

where $Q$ is a quadratic form on $\frac{1}{2} \Lambda / \Lambda$ with values in the field $\boldsymbol{F}_{2}=\{0,1\}$, then $Q$ has an Arf invariant $\Delta(Q) \in F_{2}$. It is not hard to show that $\left(V, A, e, e_{*}\right)$ has the required form only if $\Delta(Q)=0$. We leave this point to the reader.

Definition 1. A theta-function $\Theta$ on $V$ is a map $\Theta: V \rightarrow k$ satisfying

i) $\Theta(\alpha+\beta)=e_{*}(\beta / 2) \cdot e(\beta / 2, \alpha) \Theta(\alpha)$, all $\alpha \in V, \beta \in \Lambda$,

ii) $\Theta(-\alpha)=\Theta(\alpha)$, all $\alpha \in V$,

iii) $\prod_{i=1}^{4} \Theta\left(\alpha_{i}\right)=2^{-\mathrm{g}} \sum_{\eta \in \frac{1}{2} \Lambda / A} e(\gamma, \eta) \cdot \prod_{i=1}^{4} \Theta\left(\alpha_{i}+\gamma+\eta\right)$

if $\gamma=-\frac{1}{2} \sum_{1}^{4} \alpha_{i}, \alpha_{1}, \ldots, \alpha_{4} \in V$ arbitrary.

If we let

$$
S_{0}=\{\alpha \mid \Theta(\alpha) \neq 0\}=\operatorname{support}(\Theta)
$$

then $S_{0}$ is a union of cosets of $\Lambda$. The structure of $S_{0}$ is a "fine" property of $\Theta$, so we introduce:

Definition 2. The coarse support $S_{1}$ of $\Theta$ is:

$$
S_{1}=\left\{\alpha \mid \Theta(\alpha+\eta) \neq 0, \text { for some } \eta \in \frac{1}{2} \Lambda\right\}
$$

We will see in $\S 11$ that the coarse support $S_{1}$ of a theta function is either all of $V$, or $\frac{1}{2} \Lambda+W$ where $W \subset V$ is a proper subvectorspace. This is the essential difference between good and bad theta functions.

Note that $S_{0}=-S_{0}$ and $S_{1}=-S_{1}$. We always assume, in what follows, that $\Theta \neq 0$, i.e., $S_{0} \neq \phi$.

1. If $x_{1} \notin S_{1}, x_{2}, x_{3}, x_{4} \in S_{0}$, then $2 x_{1}+x_{2}+x_{3}+x_{4} \notin S_{0}$.

Proof. Use the quartic relation on $\Theta$, with $\alpha_{1}=2 x_{1}+x_{2}+x_{3}+x_{4}$, $\alpha_{2}=x_{2}, \alpha_{3}=x_{3}, \alpha_{4}=x_{4}, \gamma=-x_{1}-x_{2}-x_{3}-x_{4}$. Q.E.D.

2. $0 \in S_{1}$.

Proof. Assume $0 \notin S_{1}$. Take any $y \in S_{0}$. Apply (1.) with $x_{2}=x_{3}=y$, $x_{4}=-y$ and we get a contradiction. Q.E.D.

3. $x, y \in S_{0} \Rightarrow \frac{1}{2}(x+y) \in S_{1}$. Q.E.D.

Proof. Apply (1.) with $x_{1}=\frac{1}{2}(x+y), x_{2}=x, x_{3}=-y$ and $x_{4}=-x$. 
Because of (2.), there is an $\eta_{0} \in \frac{1}{2} \Lambda$ such that $\Theta\left(\eta_{0}\right) \neq 0$. Fix one such $\eta_{0}$. 4. $(0) \subseteq\left(S_{0}+\eta_{0}\right) \subseteq\left(2 S_{0}+\Lambda\right) \subseteq\left(4 S_{0}+\Lambda\right) \subseteq \cdots$.

Proof. By (3), if $x \in S_{0}$, then $\frac{1}{2}\left(x+\eta_{0}\right) \in S_{1}$, so $x+\eta_{0} \in 2 S_{0}+\Lambda$. This gives the $1^{\text {st }}$ inclusion. This also shows that $2 x \in 4 S_{0}+\Lambda$. Hence if $y \in 2^{k} S_{0}$, so $y=2^{k} \cdot x, x \in S_{0}$, then $2^{k} \cdot x \in 2^{k+1} S_{0}+\Lambda$. This gives the rest of the inclusions. Q.E.D.

Definition 3.

$$
S_{\infty}=\bigcup_{k \geqq 1}^{\infty}\left[2^{k} S_{0}+\Lambda\right]
$$

5. $S_{\infty}$ is a group.

Proof. Let $x, y \in S_{\infty}$. Now $x, y \in\left(2^{l} \cdot S_{0}+\Lambda\right)$ for some $l \geqq l_{0}$. Then $x=2^{l} \cdot x_{0}+\eta, y=2^{l} \cdot y_{0}+\zeta, x_{0}, y_{0} \in S_{0}$ and $\eta, \zeta \in \Lambda$. Therefore by (3), $\frac{1}{2}\left(x_{0}+y_{0}\right) \in S_{1}$, hence $2^{l}\left(x_{0}+y_{0}\right) \in 2^{l+1} \cdot S_{0}+\Lambda$. Therefore $x+y \in$ $\left(2^{l+1} S_{0}+\Lambda\right) \subset S_{\infty}$. Q.E.D.

6. $S_{\infty}=W+\Lambda$, for some subvectorspace $W \subset V$.

Proof. This is easily seen to be equivalent to asserting that $S_{\infty} / \Lambda$ is a divisible subgroup of $V / \Lambda$. But if $x \in 2^{k} \cdot S_{0}+\Lambda$, then $x=2^{k} \cdot x_{0}+\eta$, $x_{0} \in S_{0}, \eta \in \Lambda$, hence $x-\eta \in 2\left\{2^{k-1} S_{0}\right\} \subset 2 \cdot S_{\infty}$, i.e., the image of $x$ in $S_{\infty} / \Lambda$ is divisible by 2 . Q.E.D.

Definition 4. A theta function is non-degenerate if equivalently:

(a) $S_{\infty}=V$.

(a') $S_{\infty} \supset \frac{1}{2} \Lambda$.

(a") For all sufficiently large $n, 2^{n} \cdot S_{0}+\Lambda \supset \frac{1}{2} \Lambda$.

$\left(\mathrm{a}^{\prime \prime \prime}\right)$ For all sufficiently large $n$, and $\alpha \in 2^{-n-1} \Lambda$, there is an $\eta \in 2^{-n} \Lambda$ such that $\Theta(\alpha+\eta) \neq 0$.

The next step is to form, via the function $\Theta$, a sequence of graded rings:

Definition 5. If $M$ is a vector space of $k$-valued functions on $V$, let

$$
\mathscr{S}(M)=\bigoplus_{n=0}^{\infty} \mathscr{S}_{n}(M),
$$

where $\mathscr{S}_{0}(M)=k, \mathscr{S}_{1}(M)=M$, and $\mathscr{S}_{n}(M)$, for $n \geqq 2$, is the vector spac of functions on $V$ spanned by the products $f_{i_{1}} \ldots f_{i_{n}},\left(f_{i} \in M\right.$, all $\left.j\right)$. Another convenient notation is the following:

In particular, let

$$
M^{*}=\left\{\begin{array}{l}
\text { set of functions } \alpha \mapsto f(\alpha / 2), \\
\text { all } f \in M
\end{array}\right\} .
$$

$$
M_{2 k}=\text { span of the functions } \Theta_{[\beta]}, \quad \text { all } \beta \in 2^{-k} \Lambda
$$


where

$$
\Theta_{[\beta]}(\alpha)=e(\beta / 2, \alpha) \cdot \Theta(\alpha-\beta) .
$$

The corresponding rings $\mathscr{S}\left(M_{2 k}\right)$ will be the heart of our analysis. These are only half of the rings we need, however. To define the others, choose a decomposition:

$$
\Lambda=\Lambda_{1} \oplus \Lambda_{2}
$$

such that $Q_{2} \cdot \Lambda_{i}=V_{i}$ is an isotropic subspace under $e$, and such that $e_{*}(\alpha / 2)=1$ for all $\alpha \in \Lambda_{1}$ or $\Lambda_{2}$. This exists because if we choose coordinates $V \cong Q_{2}^{2 g}$ such that $\Lambda, e, e_{*}$ take their standard forms, then $\Lambda_{1}=$ $\boldsymbol{Z}_{\mathrm{g}}^{2} \times\{0\}, \Lambda_{2}=\{0\} \times \boldsymbol{Z}_{\mathrm{g}}^{2}$ have these properties. In terms of $\Lambda_{1}$ and $\Lambda_{2}$, we now define a kind of "dual" theta-function $\phi$. It is to satisfy the equations:

$$
\sum_{\zeta \in \frac{1}{2} \Lambda_{1} / \Lambda_{1}} e(\alpha, \zeta) \cdot \Theta(\alpha+\beta+\zeta) \cdot \Theta(\alpha-\beta+\zeta)=\phi(\alpha) \cdot \phi(\beta)
$$

all $\alpha, \beta \in V$. In fact, if we let $\Phi(\alpha, \beta)$ denote the left-hand side of this equation, then the quartic equations on $\Theta$ are equivalent to:

$$
\Phi(\alpha, \beta) \cdot \Phi(\gamma, \delta)=\Phi(\alpha, \delta) \cdot \Phi(\gamma, \beta)
$$

for all $\alpha, \beta, \gamma, \delta \in V$ (cf. proof of Lemma $2, \S 8$ ). This, plus the elementary fact $\Phi(\alpha, \beta)=\Phi(\beta, \alpha)$ implies that one and (up to scalars) only one such $\phi$ exists. Notice that $\phi$ satisfies the equations:

(i) $\phi(\alpha+\beta)=f_{*}(\beta) \cdot e(\beta, \alpha) \cdot \phi(\alpha)$, for all $\alpha \in V, \beta \in \frac{1}{2} \Lambda_{1}+\Lambda_{2}$, if $f_{*}\left(\frac{1}{2} \beta_{1}+\beta_{2}\right)=e\left(\frac{1}{2} \beta_{1}, \beta_{2}\right)\left(\beta_{i} \in \Lambda_{i}\right)$.

(ii) $\phi(-\alpha)=\phi(\alpha)$, all $\alpha \in V$,

as well as certain quartic equations. Now let

where

$$
M_{2 k+1}=\operatorname{span} \text { of the functions } \phi_{[\beta]}, \quad \beta \in 2^{-k-1} \cdot A
$$

$$
\phi_{[\beta]}(\alpha)=e(\beta, \alpha) \cdot \phi(\alpha-\beta) .
$$

Proposition 1. 1. $\mathscr{S}_{2}\left(M_{2 k}\right) \subseteq M_{2 k+1}$, equality holding if and only if for all $\beta \in 2^{-k-1} \Lambda, \exists \gamma \in 2^{-k} \Lambda$ such that $\phi(\beta+\gamma) \neq 0$.

2. $\mathscr{S}_{2}\left(M_{2 k+1}\right)^{*} \subseteq M_{2 k+2}$, equality holding if and only if for all $\beta \in 2^{-k-1} \Lambda, \exists \gamma \in 2^{-k} \Lambda$ such that $\Theta(\beta+\gamma) \neq 0$.

Proof. To compute $\mathscr{S}_{2}\left(M_{2 k}\right)$, note that it is spanned by the functions:

$$
f(\alpha)=\sum_{\eta \in \frac{1}{2} \Lambda_{1} / \Lambda_{1}} e\left(\eta, \frac{\beta_{1}+\beta_{2}}{2}\right) \cdot \Theta_{\left[\beta_{1}-\eta\right]}(\alpha) \cdot \Theta_{\left[\beta_{2}-\eta\right]}(\alpha)
$$


where $\beta_{i} \in 2^{-k} A$. But

$$
\begin{aligned}
f(\alpha)= & e\left(\frac{\beta_{1}+\beta_{2}}{2}, \alpha\right) \cdot \sum_{\eta \in \frac{1}{2} \Lambda_{1} / \Lambda_{1}} e\left(\alpha-\frac{\beta_{1}+\beta_{2}}{2}, \eta\right) \times \\
& \times \Theta\left(\alpha-\beta_{1}+\eta\right) \Theta\left(\alpha-\beta_{2}+\eta\right) \\
= & e\left(\frac{\beta_{1}+\beta_{2}}{2}, \alpha\right) \cdot \phi\left(\alpha-\frac{\beta_{1}+\beta_{2}}{2}\right) \cdot \phi\left(\frac{\beta_{1}-\beta_{2}}{2}\right) \\
= & \phi_{\left[\frac{\beta_{1}+\beta_{2}}{2}\right]}(\alpha) \cdot \phi\left(\frac{\beta_{1}-\beta_{2}}{2}\right) \in M_{2 k+1} .
\end{aligned}
$$

We get every $\phi_{[\gamma]}, \gamma \in 2^{-k-1} \Lambda$, in this way, if and only if every such $\gamma$ can be written:

$$
\gamma=\frac{\beta_{1}+\beta_{2}}{2}, \quad \beta_{i} \in 2^{-k} \Lambda
$$

such that

$$
\phi\left(\frac{\beta_{1}-\beta_{2}}{2}\right) \neq 0 .
$$

This is exactly the condition in (1). To prove (2), first notice the identity:

( $\alpha) \sum_{\zeta \in \frac{1}{2} \Lambda_{2} / \Lambda_{2}} e(\alpha, \zeta)^{2} \cdot \phi(\alpha+\beta+\zeta) \cdot \phi(\alpha-\beta+\zeta)$

$$
\begin{aligned}
& =\sum_{\substack{\zeta \in \frac{1}{2}, \alpha_{2} / A_{2} \\
\eta \in \mathcal{2}}} e(\alpha, \zeta)^{2} \cdot e(\alpha+\beta+\zeta, \eta) \cdot \Theta(2 \alpha+2 \zeta+\eta) \cdot \Theta(2 \beta+\eta) \\
& =\sum_{\eta \in \frac{1}{2} \Lambda_{1} / \Lambda_{1}} \Theta(2 \alpha+\eta) \cdot \Theta(2 \beta+\eta) \cdot e(\alpha+\beta, \eta) \cdot\left[\sum_{\zeta \in \frac{1}{2} \Lambda_{2} / \Lambda_{2}} e(2 \zeta, \eta)\right] \\
& =2^{\mathrm{g}} \cdot \Theta(2 \alpha) \cdot \Theta(2 \beta) \text {. }
\end{aligned}
$$

Now $\mathscr{S}_{2}\left(M_{2 k+1}\right) *$ is spanned by the various functions:

$$
f(\alpha)=\sum_{\eta \in \frac{1}{2} \Lambda_{2} / A_{2}} e\left(\eta, \beta_{1}+\beta_{2}\right) \cdot \phi_{\left[\beta_{1}-\eta\right]}(\alpha / 2) \cdot \phi_{\left[\beta_{2}-\eta\right]}(\alpha / 2)
$$

where $\beta_{i} \in 2^{-k-1} \Lambda$. But this $f$ comes out as:

$$
f(\alpha)=2^{g} \cdot \Theta_{\left[\beta_{1}+\beta_{2}\right]}(\alpha) \cdot \Theta\left(\beta_{1}-\beta_{2}\right) \in M_{2 k+2} .
$$

(2) now follows just like (1). Q.E.D.

Corollary. If $\Theta$ is non-degenerate, then for all $k \gg 0$,

$$
\begin{gathered}
\mathscr{S}_{2}\left(M_{2 k}\right)=M_{2 k+1} \\
\mathscr{S}_{2}\left(M_{2 k+1}\right)^{*}=M_{2 k+2} .
\end{gathered}
$$


Proof. The $2^{\text {nd }}$ equality is clear, by condition $\left(\mathrm{a}^{\prime \prime \prime}\right)$ of the definition of non-degenerate. As for the first, note that by formula $(\alpha)$ in the proof of the Proposition,

$$
2^{g} \Theta(\alpha)^{2}=\sum_{\zeta \in \frac{1}{2} \Lambda_{2} / \Lambda_{2}} e(\alpha, \zeta) \cdot \phi(\alpha+\zeta) \cdot \phi(\zeta) .
$$

Therefore, $[\Theta(\alpha) \neq 0] \Rightarrow\left[\phi(\alpha+\zeta) \neq 0\right.$, some $\left.\zeta \in \frac{1}{2} \Lambda_{2}\right]$. Thus the nondegeneracy of $\Theta$ implies the same for $\phi$, and the $1^{\text {st }}$ equality follows too. Q.E.D.

In the following discussion, we shall assume that $\Theta$ is non-degenerate. As usual, if $R=\Sigma R_{n}$ is a graded ring, then $R(2)$ is the graded ring $\sum R_{2 n}$. The Corollary shows that there exists a $k_{0}$ such that for all $k \geqq k_{0}$,

$$
\mathscr{S}\left(M_{k}\right)(2) \cong \mathscr{S}\left(M_{k+1}\right)
$$

In particular, the corresponding schemes

$$
X=\operatorname{Proj}\left(\mathscr{S}\left(M_{k}\right)\right),
$$

for $k \geqq k_{0}$, are all canonically isomorphic. We shall prove eventually that this $X$ is an abelian variety.

So far, we know that $\mathscr{S}\left(M_{k}\right)$ is finitely generated over $k$. Moreover, it has no nilpotents: if it did, it would have a homogeneous nilpotent element $f \in \mathscr{S}_{n}\left(M_{k}\right)$. Then $f \neq 0 \Rightarrow f(\alpha) \neq 0$, some $\alpha \in V \Rightarrow f^{N}(\alpha) \neq 0$, all $N \Rightarrow f^{N} \neq 0$ in $\mathscr{S}_{n N}\left(M_{k}\right)$. Therefore, $X$ is a reduced algebraic scheme over $k$. In fact, we can map

$$
V \mid \Lambda \rightarrow X
$$

by evaluating functions in $\mathscr{S}\left(M_{k}\right)$ at points of $V$. To be more precise, for all $\alpha \in V$, define a homogeneous prime ideal $P(\alpha) \subset \mathscr{S}\left(M_{2 k}\right)$ [resp. $\left.P(\alpha) \subset \mathscr{S}\left(M_{2 k+1}\right)\right]$ by:

resp.

$$
\begin{aligned}
P(\alpha) & =\sum_{n} P_{n}(\alpha) \\
P_{n}(\alpha) & =\left\{f \in S_{n}\left(M_{2 k}\right) \mid f\left(2^{k} \alpha\right)=0\right\} \\
& =\left\{f \in S_{n}\left(M_{2 k+1}\right) \mid f\left(2^{k} \alpha\right)=0\right\}
\end{aligned}
$$

It is easy to check that for all $k$, if the $P(\alpha)$ in $\mathscr{S}\left(M_{k}\right)$ is intersected with $\mathscr{S}\left(M_{k}\right)(2)$, the resulting ideal is equal to the $P(\alpha)$ in $\mathscr{S}\left(M_{k+1}\right)$ under the isomorphisms $(\beta)$. For this reason, we omit a $k$ in the notation $P(\alpha)$. Thus $P(\alpha)$ gives a well-defined point $\bar{P}(\alpha) \in X$. It follows easily from the definition that:

a) $\bar{P}(\alpha)$ is a k-rational point of $X$,

b) $\bar{P}(\alpha+\beta)=\bar{P}(\alpha)$, if $\beta \in \Lambda$. 
Moreover:

c) $\{\bar{P}(\alpha) \mid \alpha \in V\}$ is dense in $X$.

Proof of $c$. Take $2 k \geqq k_{0}$. If (c) were false, for large $n$, there would be a non-zero function $f \in \mathscr{S}_{n}\left(M_{2 k}\right)$ that vanished at all $\bar{P}(\alpha)$ 's. But $f(\bar{P}(\alpha))=$ $0 \Leftrightarrow f\left(2^{k} \alpha\right)=0$, so $f$ would vanish everywhere on $V$, hence $f=0$. Q.E.D.

One can do even more: for $\alpha \in V$, I claim that there is an automorphism $T_{\alpha}: X \rightarrow X$ such that $T_{\alpha}(\bar{P}(\beta))=\bar{P}(\alpha+\beta)$, all $\beta \in V$. To construct $T_{\alpha}$, let $k_{1}$ be the least integer such that $2^{k_{1}} \alpha \in \Lambda$. Define

resp.:

$$
T_{a}^{*}: \quad \mathscr{S}\left(M_{2 k}\right) \rightarrow \mathscr{S}\left(M_{2 k}\right)
$$

by:

resp.

$$
\mathscr{S}\left(M_{2 k+1}\right) \rightarrow \mathscr{S}\left(M_{2 k+1}\right)
$$

$$
\begin{aligned}
T_{\alpha}^{*} f(\beta) & =e\left(\beta, 2^{k-1} \alpha\right)^{n} \cdot f\left(\beta+2^{k} \alpha\right), & & \text { all } f \in S_{n}\left(M_{2 k}\right) \\
& =e\left(\beta, 2^{k} \alpha\right)^{n} \cdot f\left(\beta+2^{k} \alpha\right), & & \text { all } f \in S_{n}\left(M_{2 k+1}\right)
\end{aligned}
$$

(where we assume $k \geqq k_{1}$ ). To check that this is, indeed, an automorphism of $\mathscr{S}\left(M_{2 k}\right)$ [resp. $\mathscr{S}\left(M_{2 k+1}\right)$ ], it suffices to check that $T_{\alpha}^{*} \Theta_{[v]} \in M_{2 k}$, all $\gamma \in 2^{-k} \Lambda$; and $T_{\alpha}^{*} \phi_{[\gamma]} \in M_{2 k+1}$, all $\gamma \in 2^{-k-1} \Lambda$. But, in fact, one computes:

$$
\begin{aligned}
T_{\alpha}^{*} \Theta_{[\gamma]} & =e_{*}\left(2^{k-1} \alpha\right) \cdot e\left(\gamma, 2^{k} \alpha\right) \cdot \Theta_{[\gamma]} \\
T_{\alpha}^{*} \phi_{[\gamma]} & =f_{*}\left(2^{k} \alpha\right) \cdot e\left(\gamma, 2^{k+1} \alpha\right) \cdot \phi_{[\gamma]} .
\end{aligned}
$$

Moreover, one finds that $T_{\alpha}^{*}$, acting on $\mathscr{S}\left(M_{k}\right)$, induces the same automorphism on $\mathscr{S}\left(M_{k}\right)$ (2) that you get by considering the $T_{\alpha}^{*}$ acting on $\mathscr{S}\left(M_{k+1}\right)$ and carrying it across via the isomorphisms $(\beta)$ of $\mathscr{S}\left(M_{k}\right)$ (2) and $\mathscr{S}\left(M_{k+1}\right)$. Therefore, the $T_{\alpha}^{*}$ 's all define one and the same automorphism $T_{\alpha}$ of $X$. Note that:

d) $\left(T_{\alpha}^{*}\right)^{-1}(P(\beta))=P(\alpha+\beta)$.

Proof. If $f \in \mathscr{S}_{n}\left(M_{2 k}\right)$ or $\mathscr{S}_{n}\left(M_{2 k+1}\right)$, then

$T_{\alpha}^{*} f \in P(\beta) \Leftrightarrow T_{\alpha}^{*} f\left(2^{k} \beta\right)=0 \Leftrightarrow f\left(2^{k} \alpha+2^{k} \beta\right)=0 \Leftrightarrow f \in P(\alpha+\beta)$, hence

d') $T_{\alpha}(\bar{P}(\beta))=\bar{P}(\alpha+\beta)$.

One checks also (via $(\gamma)$ if you like) that:

e) $T_{\alpha_{1}+\alpha_{2}}=T_{\alpha_{1}} \circ T_{\alpha_{2}}$

f) $T_{\alpha}=$ id. $\Leftrightarrow \alpha \in \Lambda$,

so that $T$ is a faithful action of the group $V / \Lambda$ on the scheme $X$.

A remarkable consequence of all this is:

Proposition 2. If $\Theta$ is non-degenerate, then $\mathscr{S}\left(M_{k}\right)$ is an integral domain, for all $k$. 
Proof. We show first that $\mathscr{S}\left(M_{k}\right)$ is a domain if $k \geqq k_{0}$. Since $\mathscr{P}\left(M_{k}\right)$ has no nilpotents, this is equivalent to showing that $X$ is irreducible. Now $V / \Lambda$ acts on $X$, so it permutes the various components of $X$, i.e., we have a homomorphism:

$$
V \mid \Lambda \rightarrow S=\left\{\begin{array}{l}
\text { gp. of permutations } \\
\text { of components of } X
\end{array}\right\} .
$$

But $S$ is a finite group and $V / \Lambda$ is a divisible group. So $V / \Lambda$ must map each component $X_{i}$ into itself. On the other hand, the collection of points $\{\bar{P}(\alpha)\}$ forms a single orbit of the action of $V / \Lambda$ on $X$. Therefore, all these points $\{\bar{P}(\alpha)\}$ belong to a single component of $X$. Since they are also dense in $X, X$ can have only a single component. Therefore $\mathscr{P}\left(M_{k}\right)$ is a domain if $k \geqq k_{0}$.

In general, suppose some $\mathscr{S}\left(M_{k}\right)$ were not a domain. Then there would be homogeneous elements $f \in \mathscr{S}_{n}\left(M_{k}\right), g \in \mathscr{S}_{m}\left(M_{k}\right)$ such that $f \cdot g=0$, $f \neq 0, g \neq 0$. Now $f^{2}$ and $g^{2}$ can be considered as elements of $\mathscr{S}\left(M_{k+1}\right)$. Since $f \cdot g=0$, we still have $f^{2} \cdot g^{2}=0$. Also, since $\mathscr{S}\left(M_{k}\right)$ has no nilpotents, $f^{2} \neq 0$ and $g^{2} \neq 0$. Therefore $\mathscr{S}\left(M_{k+1}\right)$ is not a domain either. Continuing in this way, we find that $\mathscr{S}\left(M_{l}\right)$ is not a domain for all $l \geqq k$, which contradicts the first part of the proof. Q.E.D.

Corollary 1. The following are equivalent:

i) $\Theta$ is non-degenerate,

ii) $S_{1}=V$, i.e., for all $\alpha \in V, \exists \eta \in \frac{1}{2} \Lambda$ such that $\Theta(\alpha+\eta) \neq 0$.

iii) For all $\alpha \in \frac{1}{4} \Lambda, \exists \eta \in \frac{1}{2} \Lambda$ such that $\Theta(\alpha+\eta) \neq 0$.

Proof. Clearly (ii) $\Rightarrow$ (iii) $\Rightarrow$ (i). Now assume (i) holds. If $\Theta(\alpha+\eta)=0$, all $\eta \in \frac{1}{2} \Lambda$, then it would follow from the definition of $\phi$ that $\phi(\alpha+\beta) \times$ $\phi(\beta)=0$, all $\beta \in V$. But this means that $\phi_{[-\alpha]} \cdot \phi_{[0]}=0$, i.e., one of the rings $\mathscr{P}\left(M_{2 k+1}\right)$ is not domain. This contradicts the Prop., so (ii) must hold. Q.E.D.

Corollary 2. $\mathscr{S}\left(M_{k}\right)(2) \cong \mathscr{S}\left(M_{k+1}\right)$, for all $k \geqq 2$.

Proof. In view of Prop. 1, this follows from Cor. 1 provided that we check: $\forall \alpha \in V, \exists \eta \in \frac{1}{2} \Lambda$ such that $\phi(\alpha+\eta) \neq 0$. Looking back at the proof of the Cor. to Prop. 1, you see that this too follows from Cor. 1. Q.E.D.

To show that $X$ is actually an abelian variety, we could either define the group law explicitly, using the addition formula of $\S 2$, or else we can use only the action of $V / \Lambda$ on $X$ and combine this with general structure theorems on the automorphisms of a variety. Although the former is more elementary, we follow the latter approach as it is quicker.

$X$ is given to us together with a projective embedding. For example, $X=\operatorname{Proj}\left(\mathscr{S}\left(M_{2}\right)\right)$, so

$$
X \subset P\left(M_{2}\right)
$$


Let $L_{2}$ be the invertible sheaf induced on $X$ via this embedding. If, via the isomorphism $X \cong \operatorname{Proj}\left(\mathscr{S}\left(M_{k}\right)\right)$, we embed $X$ in $\boldsymbol{P}\left(M_{k}\right)$, the induced sheaf $L_{k}$ is just:

$$
L_{k} \cong L_{2}^{2^{k-2}}
$$

Let $\mathscr{P}$ denote the family of all invertible sheaves algebraically equivalent to $L_{2}$. We shall use the fact that Aut $(X, \mathscr{P})$, the group of automorphisms of the pair $X, \mathscr{P}$, is an algebraic group (MATSUSAKA [14], GrothenDIECK [15], p. 221-20). For all $\alpha \in V \mid \Lambda$, if $2^{k} \alpha \in \Lambda$, then $T_{\alpha}$ is induced by an automorphism $T_{\alpha}^{*}$ of $\mathscr{S}\left(M_{2 k}\right)$; therefore $T_{\alpha}^{*}\left(L_{2 k}\right) \cong L_{2 k}$; therefore $T_{\alpha}^{*}\left(L_{2}\right)$ differs from $L_{2}$ by an invertible sheaf of finite order; therefore $T_{\alpha}^{-1}(\mathscr{P})=\mathscr{P}$. In other words, the action of $V / \Lambda$ on $X$ factors through an injective homomorphism:

$$
V / \Lambda \rightarrow \operatorname{Aut}(X, \mathscr{P})
$$

Let $A$ be the Zariski-closure of $V / \Lambda$ in Aut $(X, \mathscr{P})$. Then $A$ is connected since $V / \Lambda$ is divisible and dense in $A$ (cf. proof of Prop. 2), and $A$ is commutative since $V / \Lambda$ is commutative and dense in $A$. Moreover, since the $V / \Lambda$-orbit of $\bar{P}_{0}$ is dense in $X$, the $A$-orbit of $\bar{P}_{0}$ must be an open dense set in $X$, i.e., $A$ acts generically transitively on $X$. In fact, the morphism

$$
\begin{aligned}
\psi: A & \rightarrow X \\
\sigma & \mapsto \sigma\left(\bar{P}_{0}\right)
\end{aligned}
$$

is an open immersion of $A$ in $X$. This follows since the image $\psi(A)$ is always isomorphic to $A / H, H=$ the stabilizer of $\bar{P}_{0}$; and since $A$ is commutative and acting faithfully on $X$, all stabilizers are trivial.

Next, we want to compute the dimension of $X$. I claim that the Hilbert polynomial of $\left(X, L_{2}\right)$ is given by:

Proposition 3. $\chi\left(L_{2}^{n}\right)=4^{g} \cdot n^{g}$.

Proof. For $k$ large,

$$
\begin{aligned}
\chi\left(L_{2}^{22 k}\right) & =\operatorname{dim}\left(S_{2^{2 k}}\left(M_{2}\right)\right) \\
& =\operatorname{dim}\left(M_{2+2 k}\right) .
\end{aligned}
$$

Now $M_{2(k+1)}$ is, by definition, the span of the $2^{2 g(k+1)}$ functions $\Theta_{[\beta]}$, where $\beta$ runs over cosets of $2^{-k-1} \Lambda / \Lambda$. But these functions are linearly independent. To see this, look at the automorphisms $T_{\alpha}^{*}$ of $\mathscr{S}\left(M_{2(k+1)}\right)$, where $\alpha \in 2^{-k-1} \Lambda$. Use formulae $(\gamma)$ above and note that each $\Theta_{[\gamma]}$ gives rise to a distinct set of eigenvalues for the $T_{\alpha}^{*}$ 's. Therefore, the $\Theta_{[\gamma]}$ 's could not be dependent unless one were identically zero, and this is not the case. Therefore

$$
\operatorname{dim} M_{2(k+1)}=4^{g} \cdot\left(2^{2 k}\right)^{g} .
$$


This shows that $\chi\left(L_{2}^{n}\right)$ and $4^{g} \cdot n^{g}$ agree for an infinite set of values of $n$. Since both are polynomials, they are always equal. Q.E.D.

Corollary. $\operatorname{dim} X=g$.

Returning to $A$, we find that $A$ is a commutative $g$-dimensional algebraic group containing a subgroup isomorphic to $\left(\boldsymbol{Q}_{2} / \boldsymbol{Z}_{2}\right)^{2}$. From well-known structure theorems on algebraic groups, the only such $A$ 's are abelian varieties. Therefore $A$ is complete, hence $A=X$, hence:

(I) $X$ is an abelian variety.

Moreover, in the course of proving this, we have also found that $V / \Lambda$ is acting on $X$ via translations, hence (comparing orders) we find:

(II) $\alpha \mapsto \bar{P}(\alpha)$ is a group isomorphism of $V / \Lambda$ with tor $_{2}(X)$.

Up to this point, identifying the various $\operatorname{Proj}\left(\mathscr{S}\left(M_{k}\right)\right)$ 's has been useful. But to go further, it is more convenient now to drop these identifications. Therefore, now let

$$
X_{n}=\operatorname{Proj}\left(\mathscr{P}\left(M_{2 n}\right)\right) \text {. }
$$

This is a family of isomorphic abelian varieties. However, the most natural maps between them are given by the inclusions:

$$
\begin{aligned}
M_{2 n} & \subset M_{2 n+2} \\
\mathscr{S}\left(M_{2 n}\right) & \subset \mathscr{S}\left(M_{2 n+2}\right)
\end{aligned}
$$

inducing finite morphisms:

$$
X_{n} \stackrel{p}{\longleftarrow} X_{n+1} .
$$

To check that $p$ is defined, we must know that $\mathscr{S}\left(M_{2 n+2}\right)$ is integrally dependent on $\mathscr{S}\left(M_{2_{n}}\right)$. But I claim:

$$
\Theta(\gamma)^{2} \cdot \Theta_{[\beta]}^{2}=2^{-g} \cdot \sum_{\eta \in \frac{1}{2} A / A} e(\eta, \gamma) \Theta(\eta)^{2} \cdot \Theta_{[\beta+\gamma-\eta]} \cdot \Theta_{[\beta-\gamma+\eta]} .
$$

[Proof. $\Theta(\gamma)^{2} \cdot \Theta_{[\beta]}(\alpha)^{2}=e(\beta, \alpha) \Theta(\gamma) \Theta(\gamma) \Theta(\beta-\alpha) \Theta(\alpha-\beta)$.

By the quartic relations on $\Theta$, we get

$$
\begin{aligned}
& =2^{-g} e(\beta, \alpha) \sum_{\eta} e(-\gamma, \eta) \Theta(\eta)^{2} \Theta(\beta-\alpha-\gamma+\eta) \Theta(\alpha-\beta-\gamma+\eta) \\
& \left.=2^{-g} \sum_{\eta} e(\eta, \gamma) \Theta(\eta)^{2} \cdot \Theta_{[\beta+\gamma-\eta]}(\alpha) \cdot \Theta_{[\beta-\gamma+\eta]}(\alpha) \text {. Q.E.D. }\right]
\end{aligned}
$$

Choose $\gamma \in \beta+\frac{1}{2} \Lambda$ so that $\Theta(\gamma) \neq 0$. Then if $\beta \in 2^{-n-1} \Lambda$, this equation shows that $\Theta_{[\beta]}^{2} \in \mathscr{S}\left(M_{2 n}\right)$. This proves that $p$ is a finite morphism. Since $X_{n}$ and $X_{n+1}$ are abelian varieties, $p$ must be an isogeny. 
Define prime ideals:

via

$$
\begin{aligned}
& P^{(k)}(\alpha) \subset \mathscr{S}\left(M_{2 k}\right) \\
& P^{(k)}(\alpha)=\sum_{n} P_{n}^{(k)}(\alpha) \\
& P_{n}^{(k)}(\alpha)=\left\{f \in \mathscr{S}_{n}\left(M_{2 k}\right) \mid f(\alpha)=0\right\} .
\end{aligned}
$$

Then $P^{(k)}(\alpha)$ defines a $k$-rational point $\psi_{k}(\alpha) \in X_{k}$. We have

(a) $p\left(\psi_{k+1}(\alpha)\right)=\psi_{k}(\alpha)$.

(b) $\alpha \mapsto \psi_{k}(\alpha)$ defines an isomorphism

$$
V / 2^{k} \Lambda \stackrel{\approx}{\longrightarrow} \operatorname{tor}_{2}\left(X_{k}\right) \text {. }
$$

(b) here follows from conclusion (II) above, noticing how we have reinterpreted the ideal $P(\alpha)$. In fact, if we call $X$ the common abelian variety to which all the $X_{k}$ 's were previously identified, then $\bar{P}(\alpha) \in X$ corresponds exactly to $\psi_{k}\left(2^{k} \alpha\right) \in X_{k}$. Therefore $\psi_{k}(\alpha)=0 \Leftrightarrow \bar{P}\left(2^{-k} \alpha\right)=$ $0 \Leftrightarrow 2^{-k} \alpha \in \Lambda$. Moreover, this shows that via these identifications, we get a morphism:

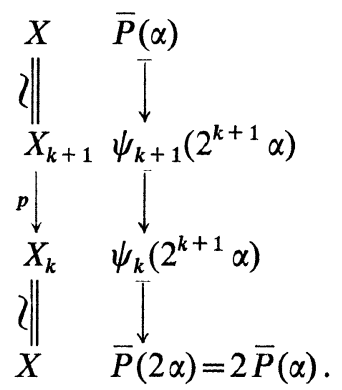

This map, from $X$ to $X$, agrees with $2 \delta$ at all points $\bar{P}(\alpha)$. Therefore it is equal to $2 \delta$. In particular:

(c) The degree of $p$ is $2^{2 g}$ and $\operatorname{Ker}(p)=\operatorname{Ker}(2 \delta)$. It follows that all the $X_{n}$ 's generate a single 2-tower. Call this $X=\left\{X_{\alpha}\right\}_{\alpha \in S}$, and let $X_{n}=$ $X_{\alpha_{n}}, \alpha_{n} \in S$. Moreover, these $\alpha_{n}$ 's are a cofinal set in $S$, by (c). In view of (a)

defines a homomorphism

$$
\alpha \mapsto\left\{\psi_{k}(\alpha)\right\}
$$

$$
\psi: V \rightarrow V(X),
$$

and (b) implies that $\psi$ is an isomorphism. More, (b) shows that the compact open subgroups $2^{k} \Lambda$ and $T\left(\alpha_{k}\right)$ correspond to each other under $\psi$.

This 2-tower is polarized too. Let $L_{k}$ be the sheaf $o(1)$ on $X_{k}$ coming from its presentation as $\operatorname{Proj}\left(\mathscr{S}\left(M_{2 k}\right)\right)$. Since the $p$ 's comes from gradation preserving homomorphisms of the $\mathscr{S}\left(M_{2 k}\right)$ 's it follows that $p^{*}\left(L_{k}\right) \cong$ $L_{k+1}$. To check that $L_{k}$ is totally symmetric, we need the inverse on $X_{k}$ : 
Let $\imath^{*}(f)(\alpha)=f(-\alpha)$, all $f \in \mathscr{S}\left(M_{2 k}\right)$.

Then $\imath^{*}$ defines an involution

such that $l\left(\psi_{k}(\alpha)\right)=\psi_{k}(-\alpha)$.

$$
\imath: X_{k} \rightarrow X_{k}
$$

Therefore $l$ agrees with the inverse of $X_{k}$ on all points $\psi_{k}(\alpha)$, hence $l=$ inverse of $X_{k}$.

Since $\imath$ is induced at all by an automorphism $\imath^{*}$ of $\mathscr{S}\left(M_{2 k}\right)$, it follows that $L_{k}$ is at least a symmetric sheaf. Since

$$
\left\{\psi_{k}(\alpha) \mid \alpha \in 2^{k-1} \Lambda / 2^{k} \Lambda\right\}=\text { Kernel of } 2 \delta \text { in } X_{k},
$$

$L_{k}$ is totally symmetric if and only if $\iota^{*}$ is the identity in $\mathscr{S}\left(M_{2 k}\right) / P^{(k)}(\alpha)$, all $\alpha \in 2^{k-1} \Lambda$. This means that for all $f \in M_{2 k}, l^{*} f-f \in P_{1}^{(k)}(\alpha)$, i.e., $f(\alpha)=$ $f(-\alpha)$. But $M_{2 k}$ is spanned by $\Theta_{[\beta]}$ 's, $\beta \in 2^{-k} \Lambda$, and if $\beta \in 2^{-k} \Lambda, \alpha \in 2^{k-1} \Lambda$, then:

$$
\Theta_{[\beta]}(-\alpha)=e\left(\frac{\beta}{2},-\alpha\right) \Theta(-\alpha-\beta)=e\left(\frac{\beta}{2}, \alpha\right) \Theta(\alpha-\beta)=\Theta_{[\beta]}(\alpha) .
$$

Therefore all the $L_{n}$ 's are totally symmetric and $\left\{X_{n}, L_{n}\right\}$ extends to a polarized 2-tower $\mathscr{T}=\left\{X_{\alpha}, L_{\alpha}\right\}$. We shall leave it to the reader to check the key fact that $\psi$ is symplectic:

(d) $e_{\lambda}(\psi \alpha, \psi \beta)=e(\alpha, \beta)$, all $\alpha, \beta \in V$.

Recapitulating this whole section so far, we have defined an arrow:

$\Xi:\left\{\begin{array}{l}\text { Given a non-degenerate } \\ \text { theta function } \Theta \text { on } V\end{array}\right\} \rightarrow\left\{\begin{array}{l}\text { construct a polarized } \\ 2 \text {-tower } \mathscr{T}=\left\{X_{\alpha}, L_{\alpha}\right\}, \\ \text { plus a symplectic isomorphism } \\ \psi: V \stackrel{\approx}{\longrightarrow} V(\underset{\leftarrow}{X})\end{array}\right\}$.

Now, on $V$ we have the vector space of functions spanned by all the $\Theta_{[\beta]}$ 's. On $V(\underset{\mathscr{X}}{X})$, we have the vector space of all theta functions $\vartheta[\Gamma(\mathscr{T})]$ of the tower $\mathscr{T}$.

Proposition 4. Via $\psi$, these vector spaces are equal:

Span of $\Theta_{[\beta]}$ 's $=\left\{\vartheta_{[s]} \circ \psi \mid s \in \Gamma(\mathscr{T})\right\}$.

Moreover, $\Theta$ itself is the unique function $f$ (up to scalars) of the form $\vartheta_{[s]} \circ \psi$ satisfying the functional equation:

$$
f(\alpha+\beta)=e_{*}(\beta / 2) \cdot e(\beta / 2, \alpha) \cdot f(\alpha), \quad \text { all } \alpha \in V, \beta \in \Lambda .
$$

Key Corollary 1. If $V=Q_{2}^{2 g}, \Lambda=Z_{2}^{2 g}$, and $e, e_{*}$ have the standard forms of $\S 9$, then $\Theta$ is exactly the theta function $\vartheta\left[\begin{array}{l}0 \\ 0\end{array}\right] \circ \psi$ associated to the 
triple $\left(X, \mathscr{T}, \psi^{-1}\right)$ in $\S 9$. In other words, $\Xi$ is an inverse to the map $\Theta$ of $\S 9$.

Proof of Prop. 4. Let $\alpha \in 2^{-k_{1}} \Lambda$ and let $k \geqq k_{1}$. Define $T_{\alpha}^{*}: \mathscr{S}\left(M_{2 k}\right) \rightarrow$ $\mathscr{S}\left(M_{2 k}\right)$ slightly differently from before:

$$
T_{\alpha}^{*} f(\beta)=e\left(\beta, \frac{\alpha}{2}\right)^{n} \cdot f(\beta+\alpha), \quad \text { all } f \in S_{n}\left(M_{2 k}\right) .
$$

Note $T_{\alpha}^{*-1}\left(P^{(k)}(\beta)\right)=P^{(k)}(\alpha+\beta)$. Let $T_{\alpha}: X_{k} \rightarrow X_{k}$ be the automorphism induced by $T_{\alpha}^{*}$. Then $T_{\alpha}\left(\psi_{k}(\beta)\right)=\psi_{k}(\alpha+\beta)$, hence $T_{\alpha}$ is translation by the point $\psi_{k}(\alpha)$, i.e.,

$$
T_{\alpha}=T_{\psi_{k}(\alpha)} .
$$

Moreover, $T_{\alpha}^{*}$ also induces a compatible isomorphism:

$$
g_{k}(\alpha): L_{k} \stackrel{\sim}{\longrightarrow} T_{\psi_{k}(\alpha)}^{*} L_{k} .
$$

For all $k \geqq k_{1}$, these are compatible, so the totality of pairs

is a point of $\mathscr{G}(\mathscr{T})$.

$$
g(\alpha)=\left\{\left(\psi_{k}(\alpha), g_{k}(\alpha) \mid k \geqq k_{1}\right\}\right.
$$

(*) $g(\alpha)=\sigma[\psi(\alpha)]$, i.e., $g(\alpha)$ is the canonical element of $\mathscr{G}(\mathscr{T})$ over the point $\psi(\alpha)$ in $V(\underline{X})$.

Proof of *. This requires checking 2 things: (i) $g(\alpha)$ is a symmetric element of $\mathscr{G}(\mathscr{T})$, i.e., $\delta_{-1} g(\alpha)=g(\alpha)^{-1}$, and (ii) $g(2 \alpha)=g(\alpha)^{2}$. In terms of $T_{\alpha}^{*}$, this is the same as:

(i) $\imath^{*} \circ T_{\alpha}^{*}=\left(T_{\alpha}^{*}\right)^{-1} \circ \imath^{*}$.

(ii) $T_{2 \alpha}^{*}=T_{\alpha}^{*} \circ T_{\alpha}^{*}$.

These are both immediate. Q.E.D.

Next, notice that $M_{2 k} \cong \Gamma\left(X_{k}, L_{k}\right)$. In fact, there is a canonical map $M_{2 k} \rightarrow \Gamma\left(X_{k}, L_{k}\right)$; it is injective, since the ring $\mathscr{S}\left(M_{2 k}\right)$ has no nilpotents, and only nilpotent elements of $\mathscr{S}_{n}\left(M_{2 k}\right)$ define trivial sections of $L_{k}^{n}$; but it is easy to check that both $\operatorname{dim} M_{2 k}$ and $\operatorname{dim} \Gamma\left(X_{k}, L_{k}\right)$ are equal to $2^{2 k g}$; therefore $M_{2 k} \cong \Gamma\left(X_{k}, L_{k}\right)$. Therefore,

$$
\Gamma(\mathscr{T})=\varliminf_{k} \Gamma\left(X_{k}, L_{k}\right) \cong \bigcup_{k} M_{2 k}=\left\{\begin{array}{l}
\text { Span of all the } \\
\text { functions } \Theta_{[\beta]} \\
\beta \in V
\end{array}\right\} .
$$

Now let $f$ be some linear combination of the $\Theta_{[\beta]}$. Say $f \in M_{2 k_{1}}$. Let $f$ define $s \in \Gamma\left(X_{k_{1}}, L_{k_{1}}\right)$. I claim that:

(*)

$$
f(\alpha)=\vartheta_{[s]}(\psi \alpha), \quad \text { all } \alpha \in V .
$$

16 Inventiones math., Vol. 3 
Taking a larger $k_{1}$ if necessary, we may suppose that $\alpha \in 2^{-k_{1}} \Lambda$. By definition, $\vartheta_{[s]}$ at $\psi \alpha$ is the "value" at the origin of $X_{k_{1}}$ of the section of $L_{k_{1}}$ obtained via the map:

$$
\Gamma\left(X_{k_{1}}, L_{k_{1}}\right) \underset{g_{k_{1}}(-\alpha)}{\sim} \Gamma\left(X_{k_{1}}, T_{\psi_{k_{1}}(-\alpha)}^{*} L_{k_{1}}\right) \underset{T_{\psi_{1}}(\alpha)}{\sim} \underset{\sim}{\sim} \Gamma\left(X_{k_{1}}, L_{k_{1}}\right) .
$$

This means that we simply apply the automorphism $\left(T_{-\alpha}^{*}\right)^{-1}$ of $M_{2 k}$ to $f$, and take the value at the origin. But $T_{-\alpha}^{*}=T_{\alpha}^{*-1}$, and $\left(T_{\alpha}^{*} f\right)(0)=f(\alpha)$, so (*) is proven. Thus the span of the $\Theta_{[\beta]}$ 's is the same as the space of functions $\vartheta_{[s]} \circ \psi, s \in \Gamma(\mathscr{T})$.

As for the final assertion of the Proposition, on the one hand, $\Theta$ does satisfy the functional equation there; and, from the general theory of the space $\vartheta[\Gamma(\mathscr{T})]$ in $\S 8$, we know that this functional equation has only a 1-dimensional set of solutions in $\vartheta[\Gamma(\mathscr{T})] \circ \psi$. Q.E.D.

Corollary 2. All g-dimensional principally polarized abelian varieties $X$ are isomorphic to Proj $\left(\mathscr{S}\left(M_{2}\right)\right)$, where $M_{2}$ is the span of the $\Theta_{[\beta]}$ 's, $\beta \in \frac{1}{2} \Lambda$, for some non-degenerate theta function $\Theta$ on $V$.

Proof. Just take $\Theta$ to be the $\vartheta\left[\begin{array}{l}0 \\ 0\end{array}\right]$ attached to $X$ as in $\S 9$, and carried over to a function on $V$ by a suitable isomorphism of $V$ and $V(X)$. Q.E.D.

Corollary 3. The open set $M_{\infty} \subset \bar{M}_{\infty}$, which in $\S 9$ represents the moduli functor $\mathscr{M}_{\infty}$, is the open set whose geometric points represent non-degenerate theta functions, i.e.,

$$
E=\left\{\begin{array}{l}
\text { set of all systems of coset representatives } \\
r: \frac{1}{4} Z_{2}^{2 g} / \frac{1}{2} Z_{2}^{2 g} \rightarrow \frac{1}{4} Z_{2}^{2 g}
\end{array}\right\} .
$$

For all $r \in E$, let

Then

$$
U_{r}=\left\{\begin{array}{l}
\text { open set in } \bar{M}_{\infty} \text { defined by } \\
X_{\alpha} \neq 0, \text { all } \alpha \in \operatorname{Image}(r)
\end{array}\right\} .
$$

$$
M_{\infty}=\bigcup_{r \in E} U_{r} .
$$

\section{\$ 11. Satake's Compactification}

In this section, I want to analyze the degenerate theta functions $\Theta$ on $V$, in the sense of $\S 10$. In particular, they all come from lower dimensional non-degenerate theta-functions via "cusps". This will show that the whole moduli scheme $\bar{M}_{\infty}$ is a disjoint union of copies of the $M_{\infty}$ 's for dimensions $g$ and lower i.e., that $\bar{M}_{\infty}$ is the Satake compactification of $M_{\infty}{ }^{1}$.

${ }^{1}$ Added in Proof. A closer study has shown that $\bar{M}_{\infty}$ is not normal along $\bar{M}_{\infty}-M_{\infty}$. Its normalization is Satake's compactification. 
Return to the discussion at the beginning of $\S 10$ : let $V, \Lambda, e, e^{*}$ be given as before. First, I want to describe a way of forming degenerate theta functions on $V$ out of theta functions on lower dimensional spaces.

Definition 1. A cusp is a subspace $W \subset V$ such that $W^{\perp} \subset W$, i.e., if $\alpha \in V$ has the property $e(\alpha, \beta)=1$, all $\beta \in W$, then $\alpha \in W$.

Given a cusp $W$, let:

$$
\begin{aligned}
\tilde{V} & =W / W^{\perp} \\
\tilde{\Lambda} & =\Lambda \cap W / \Lambda \cap W^{\perp} \\
\tilde{e} & =\text { induced skew-symmetric pairing, } \tilde{V} \times \tilde{V} \rightarrow k^{*} .
\end{aligned}
$$

Lemma. $\tilde{\Lambda}$ is a maximal isotropic lattice in $\tilde{V}$, (for $\tilde{e})$.

Proof. Notice that $\Lambda / \Lambda \cap W$ is a free $Z_{2}$-module. Therefore the sequence:

$$
0 \rightarrow \Lambda \cap W \rightarrow \Lambda \rightarrow \Lambda / \Lambda \cap W \rightarrow 0
$$

splits, and $\Lambda=\Lambda_{1} \oplus(\Lambda \cap W)$ for some sub $Z_{2}$-Module $\Lambda_{1}$. Let $V_{1}=$ $Q_{2} \cdot \Lambda_{1}$, so $V=V_{1} \oplus W$. Now I claim:

$$
(\Lambda \cap W)^{\perp}=\Lambda+W^{\perp} .
$$

[In fact, let $\alpha \in V$ satisfy $e(\alpha, \beta)=1$, all $\beta \in \Lambda \cap W$. Since $V_{1}$ and $W$ are dual vector spaces via $e$, there is a $\gamma \in W^{\perp}$ such that $e(\alpha, \beta)=e(\gamma, \beta)$ all $\beta \in V_{1}$. But then $\alpha-\gamma$ is orthogonal to both $V_{1}$ and $\Lambda \cap W$, hence orthogonal to $\Lambda$, hence $\alpha-\gamma \in \Lambda$. Thus $\alpha \in W^{\perp}+\Lambda$.]

Now to show $\tilde{\Lambda}$ is maximal isotropic, let $\alpha \in W$ have an image $\tilde{\alpha}$ in $\tilde{V}$ perpendicular to $\tilde{\Lambda}$, i.e., $\alpha \in(W \cap \Lambda)^{\perp}$. By (*), $\alpha=\alpha_{1}+\alpha_{2}$, where $\alpha_{1} \in \Lambda$, $\alpha_{2} \in W^{\perp}$. But then $\alpha_{1}=\alpha-\alpha_{2} \in W$. Therefore $\alpha_{1} \in W \cap \Lambda$ so $\tilde{\alpha}=\tilde{\alpha}_{1} \in \tilde{\Lambda}$. Q.E.D.

Definition 2. A cusp with origin is a cusp $W \subset V$, plus an element $\eta_{0} \in \frac{1}{2} \Lambda$ such that

i) $e_{*}(\alpha)=e\left(\alpha, \eta_{0}\right)^{2}$, all $\alpha \in W^{\perp} \cap\left(\frac{1}{2} \Lambda\right)$.

ii) $e_{*}\left(\eta_{0}\right)=1$.

It is not hard to check that every cusp has at least one origin: we leave this to the reader. Given a cusp with origin, look at the map

$$
\alpha \mapsto e_{*}(\alpha) \cdot e\left(\alpha, \eta_{0}\right)^{2}
$$

where $\alpha \in \frac{1}{2} \Lambda \cap W$. If $\beta \in \frac{1}{2} \Lambda \cap W^{\perp}$, then

$$
\begin{aligned}
e_{*}(\alpha+\beta) \cdot e\left(\alpha+\beta, \eta_{0}\right)^{2} & =e_{*}(\alpha) \cdot e_{*}(\beta) \cdot e(\alpha, \beta)^{2} \cdot e\left(\alpha, \eta_{0}\right)^{2} \cdot e\left(\beta, \eta_{0}\right)^{2} \\
& =e_{*}(\alpha) \cdot e\left(\alpha, \eta_{0}\right)^{2}
\end{aligned}
$$

$16^{*}$ 
Thus there is a quadratic form $\tilde{\boldsymbol{e}}_{*}: \frac{1}{2} \tilde{\Lambda} / \tilde{\Lambda} \rightarrow\{ \pm 1\}$ such that

$$
\tilde{e}_{*}(\tilde{\alpha})=e_{*}(\alpha) \cdot e\left(\alpha, \eta_{0}\right)^{2}, \quad \text { all } \alpha \in \frac{1}{2} \Lambda \cap W .
$$

It is not hard to check that the new data $\left(\tilde{V}, \tilde{\Lambda}, \tilde{e}, \tilde{e}_{*}\right)$ has the standard form required in $\S 10$ (i.e., that the associated Arf-invariant is 0 ). We leave this to the reader also.

Now let $\tilde{\Theta}$ be a theta-function on $\tilde{V}$.

Definition 3. For all $\alpha \in V$, let

$$
T_{W, \eta_{0}} \Theta(\alpha)= \begin{cases}0 \quad & \text { if } \alpha \notin \eta_{0}+W+\Lambda \\ e_{*}\left(\frac{\eta_{1}}{2}\right) e\left(\frac{\eta_{1}}{2}, \eta_{0}\right) e\left(\frac{\eta_{0}+\eta_{1}}{2}, \alpha\right) \tilde{\Theta}\left(\tilde{\alpha}_{0}\right) & \text { if } \alpha=\eta_{0}+\eta_{1}+\alpha_{0}, \eta_{1} \in \Lambda, \alpha_{0} \in W .\end{cases}
$$

Proposition 1. The above $T_{W, \eta_{0}} \tilde{\Theta}$ is well-defined (note that the $\alpha \in V$ may be decomposed in more than way as $\alpha=\eta_{0}+\eta_{1}+\alpha_{0}$ ), and is a thetafunction on $V$.

The proof of this Proposition is a ghastly but wholly straightforward set of computations. It took me several hours to do every bit and as I was no wiser at the end - except that I knew the definition was correct I shall omit details here. Our main result is:

Theorem. Let $\Theta$ be any theta-function on $V$, and let $W$ be the subspace of $V$ such that $S_{\infty}=W+\Lambda$ (cf. $\S 10$ ). Then $W$ is a cusp, and if $\eta_{0}$ is any origin for $W, \Theta$ is equal to $T_{W, \eta_{0}} \tilde{\Theta}$ for some non-degenerate thetafunction $\tilde{\Theta}$ on $\tilde{W}$. In particular, $W$ is characterized by:

$$
\text { coarse support }(\Theta)=W+\frac{1}{2} \Lambda \text {. }
$$

The proof of this theorem will be based on the $\Theta \leftrightarrow \mu$ correspondence, given in Lemma $1, \S 8$. Before taking up the proof of the Theorem, we want to give this correspondence a more intrinsic formulation. Let $V=W_{1} \oplus W_{2}$, where $W_{i}$ are maximal isotropic subspaces, such that

i) $\Lambda=\Lambda_{1} \oplus \Lambda_{2}, \Lambda_{i}=\Lambda \cap W_{i}$.

ii) $e_{*}(\alpha / 2)=1$, all $\alpha$ in $\Lambda_{1}$ or in $\Lambda_{2}$.

Then

a) Define a measure $\mu$ on $W_{1}$, from a theta function $\Theta$ on $V$ via

$$
\mu\left(\alpha_{1}+2^{n} \Lambda_{1}\right)=2^{-n g} \sum_{\alpha_{2} \in 2^{-n} \Lambda_{2} / \Lambda_{2}} e\left(\alpha_{1}, \frac{\alpha_{2}}{2}\right) \cdot \Theta\left(\alpha_{1}+\alpha_{2}\right) .
$$

b) Define a theta function $\Theta$ on $V$, from a measure $\mu$ on $W_{1}$, via

$$
\Theta\left(\alpha_{1}+\alpha_{2}\right)=e\left(\alpha_{1}, \frac{\alpha_{2}}{2}\right) \int_{\alpha_{1}+\Lambda_{1}} e\left(\alpha_{2}, \beta\right) \cdot d \mu(\beta) .
$$


Our proof will be based on the fact that any finitely additive measure $\mu$ (on the algebra of compact open subsets of $W_{1}$ ) has a support, i.e., a smallest closed set $S$ such that:

$$
\mu(U)=0, \quad \text { all compact open } U \text { 's in } W_{1}-S \text {. }
$$

Proof. Say $S_{A}$ and $S_{B}$ are closed sets such that $\mu(U)=0$ if $U \subset W_{1}-S_{A}$ or $U \subset W_{1}-S_{B}$. Then let $U \subset W_{1}-\left(S_{A} \cap S_{B}\right)$ be a compact open set. We must decompose $U$ into $U_{A} \cup U_{B}$, where $U_{A} \subset W_{1}-S_{A}$, and $U_{B} \subset$ $W_{1}-S_{B}$, and $U_{A}$ and $U_{B}$ are compact and open. For all $x \in U \cap S_{A}$, note that $x \notin S_{A}$, so we can find a compact, open neighborhood $U_{x}$ of $x$ such that

$$
U_{x} \subset U \cap\left(W_{1}-S_{B}\right) .
$$

Since $U \cap S_{A}$ is compact, it can be covered by a finite set of these $U_{x}$ 's : say

$$
U \cap S_{A} \subset\left[U_{x_{1}} \cup \cdots \cup U_{x_{n}}\right] .
$$

Let $U_{B}=U_{x_{1}} \cup \cdots \cup U_{x_{n}}$. By construction $U_{B} \subset U \cap\left(W_{1}-S_{B}\right)$ and $U_{B}$ is compact and open. Let $U_{A}=U-U_{B}$. Then $U_{A}$ is also compact and open and since $U_{B} \supset U \cap S_{B}$, it follows that $U_{A} \subset U \cap\left(W_{1}-S_{B}\right)$. By assumption on $S_{A}$ and $S_{B}$, we have $\mu\left(U_{A}\right)=0$ and $\mu\left(U_{B}\right)=0$. Therefore $\mu(U)=0$. This shows that the family of sets:

$$
\mathscr{S}=\left\{S \text { closed in } W_{1} \mid \mu(U)=0 \text { for all compact open sets } U \subset W_{1}-S\right\}
$$

is closed under finite intersections. Now let

$$
S^{*}=\bigcap_{S \in \mathscr{S}} S
$$

I claim $S^{*} \in \mathscr{S}$ too. Let $U \subset W_{1}-S^{*}$ be a compact open set. Since

$$
W_{1}-S^{*}=\bigcup_{S \in \mathscr{S}}\left(W_{1}-S\right),
$$

it follows that $U$ is covered by the open sets $U \cap\left(W_{1}-S\right)$, where $S \in \mathscr{S}$. Since $U$ is compact, it can be covered by a finite number of such open sets:

$$
U \subset\left(W_{1}-S_{1}\right) \cup \cdots \cup\left(W_{1}-S_{n}\right)
$$

where $S_{1}, \ldots, S_{n} \in \mathscr{S}$. Now let $T \in \mathscr{S}$ be a closed set contained in all these $S_{i}$. Then $U \subset W_{1}-T$. But $T \in \mathscr{S}$ means that this implies $\mu(U)=0$. So $\mu(U)=0$ whenever $U \subset W_{1}-S^{*}$, i.e., $S^{*} \in \mathscr{S}$ too. Q.E.D.

Proposition. Let $\mu$ be a non-zero even Gaussian measure on $W_{1}$ (i.e., $\mu$ has the property $(A)$ of Lemma $1, \S 8)$. Then the support $S$ of $\mu$ is a subvector space of $W_{1}$. 
Proof. Notice that if $\mu_{1}, \mu_{2}$ are 2 measures on $W_{1}$, and $\mu_{1} \times \mu_{2}$ is the induced measure on $W_{1} \times W_{1}$, then

$$
\operatorname{Support}\left(\mu_{1} \times \mu_{2}\right)=\operatorname{Support}\left(\mu_{1}\right) \times \operatorname{Support}\left(\mu_{2}\right) \text {. }
$$

Let $\xi: W_{1} \times W_{1} \rightarrow W_{1} \times W_{1}$ be the map $\xi((x, y))=(x+y, x-y)$. By definition, a Gaussian measure $\mu$ is associated to a second measure $v$ such that

$$
\xi_{*}(\mu \times \mu)=v \times v .
$$

Therefore, if $S^{\prime}=$ Support $(v)$, it follows that $\xi(S \times S)=S^{\prime} \times S^{\prime}$. In particular

$$
\begin{aligned}
\alpha \in S & \Leftrightarrow(\alpha, \alpha) \in S \times S \\
& \Leftrightarrow(2 \alpha, 0)=\zeta((\alpha, \alpha)) \in S^{\prime} \times S^{\prime} .
\end{aligned}
$$

Since $S$ is non-empty, $0 \in S^{\prime}$, and $\alpha \in S \Leftrightarrow 2 \alpha \in S^{\prime}$, i.e., $S^{\prime}=2 S$. Therefore $0 \in S$ too, and we find:

$$
\begin{aligned}
\alpha \in S & \Leftrightarrow(\alpha, 0) \in S \times S \\
& \Leftrightarrow(\alpha, \alpha)=\xi((\alpha, 0)) \in S^{\prime} \times S^{\prime} \\
& \Leftrightarrow \alpha \in S^{\prime} .
\end{aligned}
$$

Therefore $S=S^{\prime}$ also. Finally,

$$
\begin{aligned}
\alpha, \beta \in S & \Rightarrow(\alpha, \beta) \in S \times S \\
& \Rightarrow(\alpha+\beta, \alpha-\beta) \in S^{\prime} \times S^{\prime} \\
& \Rightarrow \alpha+\beta, \alpha-\beta \in S^{\prime}=S .
\end{aligned}
$$

Thus $S$ is a closed subgroup of $W_{1}$, such that $S=2 S$. Therefore $S$ is a subvectorspace over $Q_{2}$. Q.E.D.

Corollary. For all $\gamma_{2} \in W_{2}$, all theta functions $\Theta$ on $V$,

$$
\begin{array}{r}
\operatorname{Support}(\Theta) \subset\left\{\alpha \mid e\left(\alpha, \gamma_{2}\right)=1\right\} \Rightarrow \Theta\left(\alpha+\lambda \gamma_{2}\right)=e\left(\alpha, \frac{\lambda \gamma_{2}}{2}\right) \Theta(\alpha), \\
\text { all } \lambda \in Q_{2} .
\end{array}
$$

Proof. The assumption on the support of $\Theta$ implies (cf. (a) above) that $\mu\left(\alpha_{1}+2^{n} \Lambda_{1}\right)=0$ if $e\left(\alpha_{1}, \gamma_{2}\right) \neq 1$. Therefore,

$$
\operatorname{Support}(\mu) \subset\left\{\alpha_{1} \in W_{1} \mid e\left(\alpha_{1}, \gamma_{2}\right)=1\right\} \text {. }
$$

Since this support is a vector space,

$$
\operatorname{Support}(\mu) \subset W_{1} \cap\left(Q_{2} \cdot \gamma_{2}\right)^{\perp} .
$$


Let $H$ denote the hyperplane $W_{1} \cap\left(Q_{2} \cdot \gamma_{2}\right)^{\perp}$. Then

Thus

$$
\Theta\left(\alpha_{1}+\alpha_{2}\right)=e\left(\alpha_{1}, \frac{\alpha_{2}}{2}\right) \int_{\left(\alpha_{1}+\Lambda_{1}\right) \cap H} e\left(\alpha_{2}, \beta\right) \cdot d \mu(\beta) .
$$

$\Theta\left(\alpha_{1}+\alpha_{2}+\lambda \gamma_{2}\right)=e\left(\alpha_{1}, \frac{\alpha_{2}+\lambda \gamma_{2}}{2}\right) \underset{\left(\alpha_{1}+\Lambda_{1}\right) \cap H}{\int_{1}} e\left(\alpha_{2}+\lambda \gamma_{2}, \beta\right) \cdot d \mu(\beta)$

and since $e\left(\lambda \gamma_{2}, \beta\right)=1$ when $\beta \in H$, this comes out

$$
\begin{aligned}
& =e\left(\alpha_{1}, \frac{\lambda \gamma_{2}}{2}\right) \cdot\left\{e\left(\alpha_{1}, \frac{\alpha_{2}}{2}\right) \int_{\left(\alpha_{1}+\Lambda_{1}\right) \cap H} e\left(\alpha_{2}, \beta\right) \cdot d \mu(\beta)\right\} \\
& =e\left(\alpha_{1}, \frac{\lambda \gamma_{2}}{2}\right) \cdot \Theta\left(\alpha_{1}+\alpha_{2}\right) . \quad \text { Q.E.D. }
\end{aligned}
$$

In fact, I claim that the same Corollary holds for all $\gamma \in V$, not just for $\gamma \in W_{2}$. This can be seen by noting that for any $\gamma \in V$, there is a symplectic automorphism $T: V \rightarrow V$ such that $T(\Lambda)=\Lambda$, i.e., $T \in \operatorname{Sp}(V, \Lambda)$, such that $T^{-1}(\gamma) \in W_{2}$. Going back to the action of the symplectic group introduced in $\S 9$, we see that:

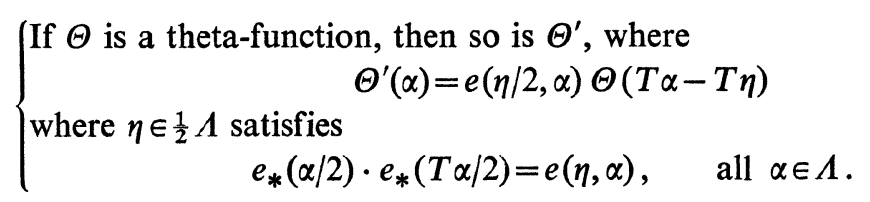

Now assume $\operatorname{Supp}(\Theta) \subset\{\alpha \mid e(\alpha, \gamma)=1\}$. Then

$$
\begin{aligned}
\operatorname{Supp}\left(\Theta^{\prime}\right) & =\eta+T^{-1}(\operatorname{Supp}(\Theta)) \\
& \subset \eta+\left\{\alpha \mid e\left(\alpha, T^{-1} \gamma\right)=1\right\} \\
& \subset\left\{\alpha \mid e\left(\alpha, 2^{n} T^{-1} \gamma\right)=1\right\} \quad(\text { if } n \gg 0) .
\end{aligned}
$$

Therefore, by the Corollary

$$
\Theta^{\prime}\left(\alpha+\lambda T^{-1} \gamma\right)=e\left(\alpha, \frac{\lambda T^{-1} \gamma}{2}\right) \Theta^{\prime}(\alpha), \quad \text { all } \lambda \in Q_{2},
$$

from which

$$
\Theta(\alpha+\lambda \gamma)=e\left(\alpha, \frac{\lambda \gamma}{2}\right) \cdot \Theta(\alpha)
$$

follows immediately. We are now ready for the Proof itself:

Proof of Theorem. We know that the support of $\Theta$ meets $\frac{1}{2} \Lambda$ (cf. $\S 10$ ): choose $\eta_{0} \in \operatorname{Supp}(\Theta) \cap \frac{1}{2} \Lambda$. Then:

$$
\operatorname{Supp}(\Theta)+\eta_{0} \subseteq W+\Lambda
$$


( $\$ 10$, assertion (4.) at the beginning). Therefore, if $\gamma \in W^{\perp} \cap(2 \Lambda)$ it follows that $e(\alpha, \gamma)=1$, all $\alpha \in \operatorname{Supp}(\Theta)$. But then by Corollary above - as generalized -

$$
\Theta(\alpha+\lambda \cdot \gamma)=e\left(\alpha, \frac{\lambda \gamma}{2}\right) \cdot \Theta(\alpha), \quad \text { all } \lambda \in Q_{2}
$$

This shows that

$$
\Theta(\alpha+\gamma)=e\left(\alpha, \frac{\gamma}{2}\right) \cdot \Theta(\alpha), \quad \text { all } \gamma \in W^{\perp}
$$

In particular, $\Theta\left(\eta_{0}+\gamma\right) \neq 0$, all $\gamma \in W^{\perp}$, hence $W^{\perp}+\eta_{0} \subseteq W+\Lambda+\eta_{0}$. Therefore $W^{\perp} \subseteq W$, i.e., $W$ is a cusp.

Now suppose we take an arbitrary point $\alpha$ in the Support of $\Theta$. We know that $\alpha$ can be written as:

But then:

$$
\alpha=\eta_{0}+\eta_{1}+\alpha_{0}, \quad \eta_{1} \in \Lambda, \alpha_{0} \in W .
$$

$$
\begin{aligned}
\Theta(\alpha) & =e_{*}\left(\frac{\eta_{1}}{2}\right) \cdot e\left(\frac{\eta_{1}}{2}, \eta_{0}+\alpha_{0}\right) \cdot \Theta\left(\eta_{0}+\alpha_{0}\right) \\
= & e_{*}\left(\frac{\eta_{1}}{2}\right) \cdot e\left(\frac{\eta_{1}}{2}, \eta_{0}\right) \cdot e\left(\frac{\eta_{0}+\eta_{1}}{2}, \alpha\right) \cdot\left[e\left(\alpha, \frac{\eta_{0}}{2}\right) \cdot \Theta\left(\eta_{0}+\alpha\right)\right] .
\end{aligned}
$$

Define a function $\tilde{\Theta}$ on $W$ by

$$
\tilde{\Theta}(\alpha)=e\left(\alpha, \frac{\eta_{0}}{2}\right) \cdot \Theta\left(\alpha+\eta_{0}\right)
$$

If $\gamma \in W^{\perp}$, we compute (using $\left(^{*}\right)$ ):

$$
\begin{aligned}
\tilde{\Theta}(\alpha+\gamma) & =e\left(\alpha+\gamma, \frac{\eta_{0}}{2}\right) \cdot \Theta\left(\alpha+\eta_{0}+\gamma\right) \\
& =e\left(\gamma, \frac{\eta_{0}}{2}\right) \cdot e\left(\alpha+\eta_{0}, \frac{\gamma}{2}\right) \cdot e\left(\alpha, \frac{\eta_{0}}{2}\right) \cdot \Theta\left(\alpha+\eta_{0}\right) \\
& =\tilde{\Theta}(\alpha) .
\end{aligned}
$$

This shows that $\tilde{\Theta}$ is, in reality, a function on $\tilde{V}=W / W^{\perp}$, and that $\Theta$ is exactly the function $T_{W, \eta_{0}} \tilde{\Theta}$ obtained from $\tilde{\Theta}$ via Definition 3 .

To check that $\eta_{0}$ is an origin for $W$, look at $\left(^{*}\right)$ when $\gamma^{\perp} \in W \cap \Lambda$. Then:

hence

$$
e\left(\alpha, \frac{\gamma}{2}\right) \cdot \Theta(\alpha)=\Theta(\alpha+\gamma)=e_{*}\left(\frac{\gamma}{2}\right) \cdot e\left(\frac{\gamma}{2}, \alpha\right) \cdot \Theta(\alpha)
$$

$$
e_{*}\left(\frac{\gamma}{2}\right)=e(\alpha, \gamma) \quad \text { if } \Theta(\alpha) \neq 0
$$


So

$$
e_{*}\left(\frac{\gamma}{2}\right)=e\left(\eta_{0}, \gamma\right), \quad \text { all } \gamma \in W^{\perp} \cap \Lambda
$$

Moreover, using

and

$$
\Theta\left(\eta_{0}\right)=\Theta\left(-\eta_{0}+2 \eta_{0}\right)=e_{*}\left(\eta_{0}\right) \Theta\left(-\eta_{0}\right)
$$

$$
\Theta\left(-\eta_{0}\right)=\Theta\left(\eta_{0}\right) \neq 0,
$$

we conclude that $e_{*}\left(\eta_{0}\right)=1$ too.

The fact that $\tilde{\Theta}$ is again a theta-function is simply a matter of applying the calculations of Prop. 1 in reverse and is quite straightforward. We omit this. The final point is that $\tilde{\Theta}$ is non-degenerate. But since $S_{\infty} \supseteq W$, we know that for all $\alpha \in W, \alpha=2^{k} \beta+\eta_{1}$, where $\Theta(\beta) \neq 0, \eta_{1} \in \Lambda$. Then $\beta=\eta_{0}+\eta_{2}+\beta_{0}, \eta_{2} \in \Lambda, \beta_{0} \in W$, and $\widetilde{\Theta}\left(\beta_{0}\right) \neq 0$. Since

$$
\alpha-2^{k} \beta_{0}=\eta_{1}+2^{k} \eta_{0}+2^{k} \eta_{2} \in W \cap \Lambda,
$$

this shows that for all $\alpha \in W, \alpha=2^{k} \beta_{0}+\eta_{3}$, where $\tilde{\Theta}\left(\beta_{0}\right) \neq 0, \eta_{3} \in W \cap \Lambda$. This means exactly that the $S_{\infty}$ for $\widetilde{\Theta}$ is all of $\tilde{V}$, i.e., $\widetilde{\Theta}$ is non-degenerate. Q.E.D.

The main Theorem can now be reformulated to give a Satake-like decomposition of $\bar{M}_{\infty}$. More precisely, for each integer $g \geqq 0$, let $\bar{M}_{\infty}(g)=$ the Proj defined in $\S 9$, Def. 3 with indices $\alpha \in Q_{2}^{2 g}$. $M_{\infty}(g)=$ the open set in $\bar{M}_{\infty}(g)$ whose geometric points are the nondegenerate theta functions.

If $h<g$, we define a vast number of closed immersions

$$
i_{W}: \bar{M}_{\infty}(h) \rightarrow \bar{M}_{\infty}(g)
$$

as follows: let $W \subseteq Q_{2}^{2 g}$ be a cusp such that $2 h=\operatorname{dim}\left(W / W^{\perp}\right)$. For each such $W$, choose an origin $\eta_{0} \in \frac{1}{2} Z_{2}^{2 g}$, and a symplectic isomorphism:

such that

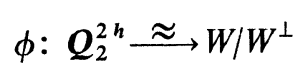

$$
\begin{gathered}
\phi\left(\boldsymbol{Z}_{2}^{2 h}\right)=W \cap \Lambda / W^{\perp} \cap \Lambda, \\
\chi\left(\frac{1}{2}^{t} a_{1} \cdot a_{2}\right)=\tilde{e}_{*}\left(\frac{1}{2} \phi(a)\right), \quad \text { all } a \in \boldsymbol{Z}_{2}^{2 h} .
\end{gathered}
$$

Then $i_{W}$ is defined by the homomorphism of the homogeneous coordinate ring:

$$
i_{W}^{*}\left(X_{\alpha}^{(\mathrm{g})}\right)= \begin{cases}0 & \text { if } \alpha \notin \eta_{0}+W+Z_{2}^{2 g} \\ e_{*}\left(\frac{\eta_{1}}{2}\right) e\left(\frac{\eta_{1}}{2}, \eta_{0}\right) e\left(\frac{\eta_{0}+\eta_{1}}{2}, \alpha\right) \cdot X_{\phi^{-1}\left(\alpha_{0}\right)}^{(h)} \\ \text { if } \alpha=\eta_{0}+\alpha_{0}+\eta_{1}, \alpha_{0} \in W, \eta_{1} \in Z_{2}^{2 g} .\end{cases}
$$


(Here $X_{\alpha}^{(g)}, X_{\alpha}^{(h)}$ are the coordinates used to define $\bar{M}_{\infty}(g), \bar{M}_{\infty}(h)$ respectively). Then we get the restatement:

\section{Main Theorem.}

$$
\bar{M}_{\infty}(g)=\left\{\begin{array}{l}
\text { disjoint union of the locally } \\
\text { closed subschemes } i_{W}\left(M_{\infty}(h)\right)
\end{array}\right\},
$$

the union being taken over all cusps $W \subseteq Q_{2}^{2}$.

\section{$\S 12$. Analytic Theta Functions}

In this section, we work over the field $\boldsymbol{C}$ of complex numbers. We have 2 purposes: (a) to sketch an approach to the classical theory of $\Theta$-functions, analogous to our theory of algebraic $\Theta$-functions, and (b) to use this to compute our algebraic $\Theta$-functions via the classical ones, when $k=C$.

We will make use of the following lemma:

Lemma 1. Let $X$ be a compact Kähler manifold. Then the operator

defines a surjection:

$$
\frac{1}{2 \pi i} \partial \bar{\partial}
$$

$$
\left\{\begin{array}{l}
C^{\infty} \text { real } \\
\text { functions on } X
\end{array}\right\} \rightarrow\left\{\begin{array}{l}
\text { real closed } C^{\infty}(1,1) \text {-forms } \Omega \text { on } X, \\
\text { with } 0 \text { cohomology class }
\end{array}\right\}
$$

with kernel consisting only of constants.

Corollary. Let $\mathbf{L}$ be an analytic line bundle on $X$. Let $c_{1}(L) \in H^{2}(X, C)$ be its first chern class. Then for all real closed $C^{\infty}(1,1)$-forms $\Omega$ whose cohomology class equals $c_{1}(L)$, there is one and (up to a constant) only one Hermitian structure \|\| on $\boldsymbol{L}$ whose associated curvature form is $\Omega$.

The lemma is standard and we omit the proof. The Corollary can be proven by choosing one Hermitian structure \|\|$_{0}$ on $L$ : let $\Omega_{0}$ be its curvature form. Then any other Hermitian structure on $\boldsymbol{L}$ is given by $\rho \cdot\|\|_{0}$, where $\rho$ is a positive real $C^{\infty}$ function on $X$ : and its curvature form $\Omega$ is

$$
\Omega=\frac{1}{2 \pi i} \partial \bar{\partial} \log \rho+\Omega_{0} .
$$

Now use the Lemma and everything comes out. Q.E.D.

In particular, when $X$ is an abelian variety, an analytic line bundle $L$ on $X$ has one and (up to a constant) only one Hermitian structure \| $\|$ whose curvature form $\Omega$ is a translation-invariant $(1,1)$-form. In what follows, we will always put this Hermitian structure on line bundles on abelian varieties. In this case, $\Omega$ is determined by its value at the origin. 
Now let $\hat{X}$ be the universal covering space of $X . \hat{X}$ is a complex vector space, and if

$$
p: \hat{X} \rightarrow X
$$

is the canonical homomorphism, dp induces a canonical identification between $\hat{X}$ and the tangent space of $X$ at the origin (or at any other point). Therefore, any translation-invariant real 2-form $\Omega$ on $X$ defines and is defined by a real-linear skew-symmetric form:

$$
E: \hat{X} \times \hat{X} \rightarrow \boldsymbol{R} .
$$

$E$ is a $(1,1)$-form if and only if $E(i x, i y)=E(x, y)$, all $x, y \in X$. Moreover, let $\Lambda=\operatorname{kernel}(p) . \Lambda$ is a lattice in $X$, canonically isomorphic to $H_{1}(X, Z)$. Since the first chern class of a line bundle is integral, if $E$ represents $c_{1}(L)$, then $E$ must take integral values on $\Lambda \times \Lambda$ :

$$
E(\Lambda \times \Lambda) \subseteq Z
$$

If we lift $\boldsymbol{L}$ to $\hat{X}$, we have a situation in which the following lemma applies:

Lemma 2. Let $Y$ be a complex vector space, and let $L_{1}, L_{2}$ be 2 analytic-Hermitian line bundles on $Y$. Then a holomorphic-unitary isomorphism $\phi: L_{1} \stackrel{\sim}{\longrightarrow} L_{2}$ exists if and only if the curvature forms of $L_{1}, L_{2}$ are equal; if so, $\phi$ is unique up to a scalar of absolute value 1.

Proof. Standard methods.

In particular, let $Y=\hat{X}$, and let $\boldsymbol{M}=p^{*}(\boldsymbol{L})$ be induced from an abelian variety. Give $\boldsymbol{L}$ and hence $\boldsymbol{M}$ the Hermitian structure with constant curvature form $E$. The above lemma has 2 applications:

(I) Construction of a nilpotent group $\mathscr{G}$ : If $x \in X$, and $T_{x}$ denotes translation by $x$, then the lemma shows that $M$ and $T_{x}^{*} M$ are holomorphic-unitary isomorphic. If

$$
\mathscr{G}(\boldsymbol{M})=\left\{(x, \Phi) \mid \Phi \text { a holo.-unit. isom. of } \boldsymbol{M} \text { with } T_{x}^{*} M\right\},
$$

then $\mathscr{G}(\boldsymbol{M})$ is, as before, a group lying in an exact sequence:

$$
1 \rightarrow C_{1}^{*} \rightarrow \mathscr{G}(M) \rightarrow X \rightarrow 0
$$

$\left(C_{1}^{*}=\right.$ complex numbers of absolute value 1$)$.

(II) Construction of canonical "trivialization" of $\boldsymbol{M}$ : Let 1 denote the trivial analytic line bundle over $X$ with canonical section 1 . To put a Hermitian structure on 1 , we may set $\|1\|=$ any positive real $C^{\infty}$ function. For example, let

$$
\|1\|(x)=e^{-\pi / 2 H(x, x)}
$$


where $H$ is a Hermitian form on $X$. The corresponding curvature form $E: \hat{X} \times \hat{X} \rightarrow \boldsymbol{R}$ is easily checked to equal $\operatorname{Im}(H)$. But

sets up an isomorphism:

$$
H \mapsto E=\operatorname{Im}(H)
$$

$$
\left\{\begin{array}{l}
\text { hermitian } \\
\text { forms on } X
\end{array}\right\} \stackrel{\sim}{\longrightarrow}\left\{\begin{array}{l}
\text { real skew-symmetric forms } E \text { on } X \\
\text { such that } E(i x, i y)=E(x, y)
\end{array}\right\},
$$

so for each $\boldsymbol{L}$ on $X$ with translation-invariant curvature form, we have a unique Hermitian structure on $\mathbf{1}$ of the above type so that $\mathbf{1} \cong \boldsymbol{L}$. In particular, we get a canonical

$$
1 \cong M .
$$

We can now develop a theory along similar lines to our algebraic theory. For example, if $H$ is positive definite, then let:

$\mathscr{H}=$ Hilbert space of $L^{2}$-holomorphic sections of $\boldsymbol{M}$ over $\hat{X}$.

Then $\mathscr{G}(\boldsymbol{M})$ has a natural unitary representation on $\mathscr{H}$, it is irreducible, and it turns out to be the only irreducible unitary representation of $\mathscr{G}(\boldsymbol{M})$ in which $\boldsymbol{C}_{1}^{*} \subset \mathscr{G}(\boldsymbol{M})$ acts by its natural character. This is the situation described by CARTIER [2], and studied by CARTIER and many others, e.g., MACKEY, FoCK, WeIL etc. Exactly as in $\S 1, \mathscr{G}(\boldsymbol{M})$ governs the "descent" of the Hermitian bundle $M$ to the abelian variety $X$, (or to other ones $X^{\prime}=[\hat{X} /$ another lattice $]$ ), and the "descent" of holomorphic sections of $\boldsymbol{M}$ to holomorphic sections of its descended form. Thus we get:

Proposition 1. There is a $1-1$ correspondence between

1. Hermitian-analytic line bundles $\boldsymbol{L}^{\prime}$ on $X$ such that $p^{*} \boldsymbol{L}^{\prime} \cong \boldsymbol{M}$,

2. subgroups $K \subset \mathscr{G}(\boldsymbol{M})$, such that $K \cap C_{1}^{*}=\{1\}$ whose image in $\hat{X}$ is $\Lambda=\operatorname{ker}(p: \hat{X} \rightarrow X)$.

Moreover, the holomorphic sections of $M$ of the form $p^{*}\left(s^{\prime}\right), s^{\prime} \in \Gamma\left(X, L^{\prime}\right)$, are exactly those sections $s$ which are invariant under $K$, i.e.,

$$
s=T_{-x}^{*}(\phi(s)), \quad \text { all }(x, \phi) \in K .
$$

Proof. Straightforward.

Finally, via the canonical trivialization of $\boldsymbol{M}$, holomorphic sections of $\boldsymbol{M}$ correspond to holomorphic functions on $\hat{X}$ : thus each section $s \in \Gamma(X, L)$ defines a holomorphic function on $\hat{X}$. These are the classical theta-functions.

As far as moduli are concerned, the simplest and most basic result is the following: we set out to classify triples consisting of - 
1. a complex vector space $Y$, of dimension 2;

2. an analytic, Hermitian line bundle $\boldsymbol{M}$ on $Y$, with curvature form $E=\operatorname{Im} H, H$ positive definite.

3. Parametrized lattices in $Y$, i.e., monomorphisms

such that

$$
\alpha: Z^{2 g} \rightarrow Y
$$

if

$$
E(\alpha x, \alpha y)={ }^{t} x_{1} \cdot y_{2}-{ }^{t} x_{2} \cdot y_{1}
$$

$$
x=\left(x_{1}, x_{2}\right), \quad y=\left(y_{1}, y_{2}\right) .
$$

Such triples arise if we start with a principally polarized abelian variety $(X, L)$, together with a symplectic isomorphism:

$$
\beta: Z^{2 g} \stackrel{\sim}{\longrightarrow} H_{1}(X, Z)
$$

Namely, let $Y=\hat{X}, \boldsymbol{M}=p^{*} \boldsymbol{L}$ with canonical Hermitian structure, and let $\beta$ define $\alpha$ via the natural maps $H_{1}(X, Z) \cong \operatorname{Ker}(p: \hat{X} \rightarrow X) \subset \hat{X}$. Conversely, the triple $(Y, M, \alpha)$ determines $X$ and $\beta$, and $L$ up to replacing $L$ by $T_{x}^{*} L$, some $x \in X$.

Let $\mathfrak{H}=$ SIEGEL's $g \times g$ upper half-plane. Then the moduli result is:

Proposition 2. There is a natural bijection between the set of isomorphism classes of triples $(\boldsymbol{Y}, \boldsymbol{M}, \alpha)$ and $\mathfrak{H}$. In this bijection, $\tau \in \mathfrak{H}$ corresponds to

$$
\begin{aligned}
& Y=C^{g} \\
& M=1 \quad \text { with hermitian structure }\|1\|(x)=e^{-\frac{\pi}{2} t_{x} \cdot B \cdot \bar{x}} \\
& \alpha\left(\left(x_{1}, x_{2}\right)\right)=x_{1}+\tau \cdot x_{2}
\end{aligned}
$$

where $B=(\operatorname{Im} \tau)^{-1}$.

The final topic I want to discuss is the relation between the classical and algebraic theories. Let's start with:

$X \quad=$ abelian variety;

$L \quad=$ symmetric, ample, degree 1 sheaf on $X$. [Assume for simplicity that $L$ is so chosen among its translates $T_{x}^{*} L, x \in X_{2}$, that its unique section is even; equivalently, that the Arf invariant of $Q$,

Let where $e_{*}^{L}(x)=(-1)^{Q(x)}$, is 0 .]

$\boldsymbol{L} \quad=$ line bundle on $X$ whose holomorphic sections are $L$;

$\hat{X} \quad=$ universal covering space of $X$;

$V_{2}(X)=2$-Tate group of $X$. 
Also, let $A_{2}=$ inverse image in $\hat{X}$ of $\operatorname{tor}_{2}(X)$, i.e.,

$$
\bigcup_{n} 2^{-n} \cdot \Lambda, \text { if } \Lambda=\operatorname{Ker}(p: \ddot{X} \rightarrow X) .
$$

Then we have canonical maps:

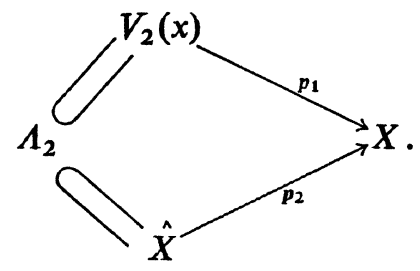

Note that $\Lambda_{2}$ is dense in both $V_{2}(X)$ and $X$. We have "trivialized" $\boldsymbol{L}$ when it is pulled up to $V_{2}(X)$ or to $X$, in $\S 8$ and just above. Thus we have 2 distinct trivializations of $L$ on $A_{2}$. The main result is that these differ by an elementary factor:

Theorem 3. Let 1 denote the trivial complex line bundle on $\Lambda_{2}$. Then the following diagram commutes:

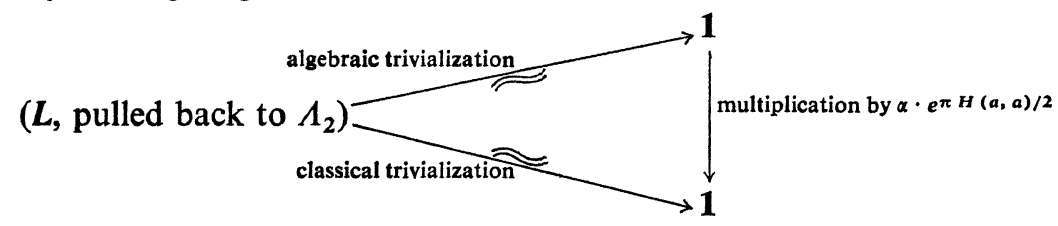

where $\alpha \in C^{*}$ and $E=\operatorname{Im}(H)$ is the curvature form of $\boldsymbol{L}$.

Proof. Let $\boldsymbol{M}_{i}=p_{i}^{*} \boldsymbol{L}=$ induced line bundle on $V_{2}(X)$ or $\hat{X}$. Let $\psi$ : $M_{2} \underset{\approx}{\approx}$ be the classical trivialization. The algebraic trivialization of $\boldsymbol{M}_{1}$ is based on finding a distinguished collection of isomorphisms

$$
\varphi_{a}: M_{1} \rightarrow T_{a}^{*} M_{1},
$$

all $a \in V_{2}(X)$. In fact, let $l=$ inverse map in all our groups, and let $\rho$ : $M_{i} \stackrel{\sim}{\sim} l^{*} M_{i}$ be the isomorphism induced by the symmetry of $L$. Then, for all elements $2 a \in V_{2}(X), \varphi_{2 a}$ is characterized by the existence of $\varphi_{a}$ satisfying:

i) $\varphi_{2 a}=T_{a}^{*} \varphi_{a} \circ \varphi_{a}$,

ii) $\imath^{*} \varphi_{a} \circ \rho=T_{-a}^{*}\left[\rho \circ \varphi_{a}^{-1}\right]$,

iii) $\varphi_{a}$ is induced by an algebraic isomorphism

$$
\varphi_{a}^{\prime}:\left(2^{n} \delta\right)^{*} L \stackrel{\sim}{\longrightarrow}\left(2^{n} \delta\right)^{*}\left(T_{p_{1}(a)}^{*} L\right)
$$


for some $n$, i.e., via the factorization:

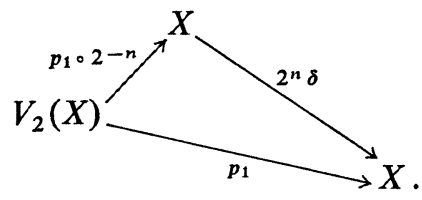

But introduce, for all $a \in X$, isomorphisms $\psi_{a}$ from $\boldsymbol{M}_{2}$ to $T_{a}^{*} \boldsymbol{M}_{2}$ via:

$$
M_{2} \underset{\psi}{\approx} \mathbf{~} 1 \underset{\text { mult. by } f_{a}(x)}{\approx} T_{a}^{*} \mathbf{1} \underset{T_{a}^{*} \psi}{\stackrel{\approx}{\approx}} T_{a}^{*} M
$$

where

Also introduce

$$
f_{a}(x)=e^{\pi[H(x, a)+H(a, a) / 2]} .
$$

$$
\rho^{\prime}: M_{2} \underset{\psi}{\underset{\text { canonical identification }}{\rightleftharpoons}} l^{*} 1 \underset{\imath^{*} \psi}{\underset{\approx}{\approx}} l^{*} M .
$$

One checks easily that $\psi_{a}$ and $\rho^{\prime}$ are holomorphic and unitary isomorphisms. Therefore $\rho$ and $\rho^{\prime}$ can differ only by a constant: and since both are the identity at $0 \in X, \rho=\rho^{\prime}$. Moreover, if $a \in 2^{-n} \Lambda$, then the algebraic isomorphism $\varphi_{a}^{\prime}:\left(2^{n} \delta\right)^{*} \boldsymbol{L} \stackrel{\sim}{\longrightarrow}\left(2^{n} \delta\right)^{*} T_{p_{2}(a)}^{*} \boldsymbol{L}$, referred to in (iii) above, induces an isomorphism $\varphi_{a}^{\prime \prime}: M_{2} \rightarrow T_{a}^{*} M_{2}$ via the factorization

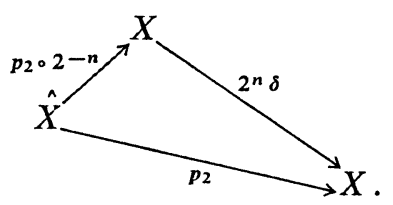

Since $\varphi_{a}^{\prime \prime}$ is also holomorphic and unitary, it differs from $\psi_{a}$ only by a constant. Next, note that $\left\{f_{a}\right\}$ satisfy the identities:

$\left.\mathrm{i}^{\prime}\right) f_{2 a}(x)=f_{a}(x+a) \cdot f_{a}(x)$

ii') $f_{a}(-x)=f_{a}(x-a)^{-1}$.

These translate readily into the identities on the $\left\{\psi_{a}\right\}$ :

$\left.\mathrm{i}^{\prime \prime}\right) \psi_{2 a}=T_{a}^{*} \psi_{a} \circ \psi_{a}$.

ii') $\imath^{*} \psi_{a} \circ \rho=T_{-a}^{*}\left[\rho \circ \psi_{a}^{-1}\right]$.

Finally, $\mathrm{i}^{\prime \prime}, \mathrm{ii}^{\prime \prime}$, plus the fact that $\varphi_{a}^{\prime}$ induces $\psi_{a}$, shows that $\psi_{a}$ and $\varphi_{a}$ induce the same isomorphism of $L$ on $\Lambda_{2}$, with $T_{a}^{*}\left(L\right.$ on $\left.\Lambda_{2}\right)$, all $a \in \Lambda_{2}$.

Finally, to compare the 2 trivializations, start with the unit section 1 of 1 on $\Lambda_{2}$. This goes over, via the algebraic trivialization, to $a$ section $s$ of $L$ on $\Lambda_{2}$ such that, for all $a \in \Lambda_{2}$,

$$
s(a)=\phi_{a}(0)[s(0)]
$$


(i.e., $\phi_{a}(0)$ is the induced isomorphism from the fibre $\boldsymbol{L}_{0}$ or $\left(\boldsymbol{M}_{1}\right)_{0}$ to the fibre $\boldsymbol{L}_{p_{1}(a)}$ or $\left.\left(\boldsymbol{M}_{1}\right)_{a}\right)$ But under the classical trivialization $\psi, \psi_{a}(0)$ corresponds to the isomorphism of fibres:

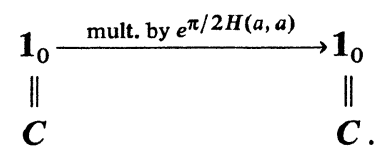

Therefore, the section $s$ goes over, under the classical trivialization, to a section of 1 which, if it has value $\alpha$ at 0 , has value

$$
\alpha \cdot e^{\pi / 2 H(a, a)}
$$

at $a$. All in all, the section 1 of $\mathbf{1}$ has gone into the section

of 1. Q.E.D.

$$
g(a)=\alpha \cdot e^{\pi / 2 H(a, a)}
$$

Corollary. If the unique section $s$ of $\boldsymbol{L}$ (up to scalars) defines

a) the holomorphic function $\Theta_{h}$ on $\hat{X}$ via the classical trivialization,

b) the 2-adic theta-function $\Theta_{a}$ on $V_{2}(X)$ via the algebraic trivialization, then

all $x \in \Lambda_{2}$.

$$
\Theta_{h}(x)=\alpha \cdot e^{\frac{\pi}{2} H(x, x)} \cdot \Theta_{a}(x)
$$

To calculate $\Theta_{h}$ and hence $\Theta_{a}$ by analytic means, we must know the "descent data"

$$
K \subset \mathscr{G}\left(M_{2}\right)
$$

that defines $L$ on $X$. Let $e_{*}: \frac{1}{2} \Lambda / \Lambda \rightarrow\{ \pm 1\}$ be the quadratic character defined by $L$. Then, as we saw in $\S 8$, the descent data for the pull-back $M_{1}$ of $L$ is the group:

$$
\left\{(x, \phi) \mid x \in \Lambda \cdot Z_{2}, \phi=e_{*}\left(\frac{1}{2} x\right) \cdot \phi_{x}\right\} .
$$

In view of the proof of the theorem, this implies that

$$
K=\left\{(x, \psi) i x \in \Lambda, \psi=e_{*}\left(\frac{1}{2} x\right) \cdot \psi_{x}\right\}
$$

(Notation as in proof of Theorem). Now a $K$-invariant section $s$ of $\boldsymbol{M}_{2}$ is one which satisfies $T_{\alpha}^{*}(s)=\phi(s)$, all $(a, \phi) \in K$. Going back to the definition of $\psi_{a}$, one sees that if $f=\psi(s)$ is the function on $\hat{X}$ corresponding to $s$, then $f$ is $K$-invariant if and only if

$$
f(x+a)=e_{*}\left(\frac{1}{2} a\right) f_{a}(x) \cdot f(x)
$$

all $x \in \hat{X}, a \in \Lambda$. From this it follows that $\Theta_{h}$ must be the unique holomorphic function satisfying (*). 
To go further and write down this $\Theta_{h}$ as an infinite series, it is convenient to introduce coordinates. Let

$$
i: Z^{2 g} \approx \Lambda \quad \text { be a symplectic isomorphism. }
$$

Coordinatize $\hat{X}$ via

$$
\dot{X} \cong C^{g}
$$

so that $i\left(\left(n_{1}, 0\right)\right)=n_{1}$, and let $\tau$ be the $g \times g$ matrix defined by

$$
i\left(\left(0, n_{2}\right)\right)=\tau \cdot n_{2} \text {. }
$$

Because of our assumption on $e_{*}^{L}$, hence on $e_{*}$, if we choose coordinates correctly, we can assume that

$$
e_{*}\left[\frac{1}{2} i\left(n_{1}, n_{2}\right)\right]=(-1)^{t_{n_{1}} \cdot n_{2}} .
$$

As we saw in Prop. 2, if we now express:

$$
H(z, z)={ }^{t} z \cdot B \cdot \bar{z}
$$

then $B=(\operatorname{Im} \tau)^{-1}$. Finally, set

$$
\Theta_{h}(z)=e^{\frac{\pi}{2} t_{z} \cdot B \cdot z} \cdot \sum_{n \in Z g} e^{2 \pi i\left[\frac{1}{2} t_{n} \cdot \tau \cdot n+t_{n} \cdot z\right]} .
$$

It is easy to check that this is a holomorphic function satisfying $(*)$. Therefore, this is the sought-for theta-function. Combining this with the Corollary, we find

$$
\Theta_{a}(z)=e^{\frac{\pi}{2} t_{z} \cdot B \cdot(z-\bar{z})} \cdot \sum_{n \in Z^{g}} e^{2 \pi i\left[\frac{1}{2} t_{n} \cdot \tau \cdot n+t_{n} \cdot z\right]} \quad \text { all } z \in \bigcup_{k} 2^{-k} \Lambda
$$

If

$$
z=i\left(\left(\alpha_{1}, \alpha_{2}\right)\right), \quad \alpha_{i} \in \bigcup_{k} 2^{-k} \cdot\left(Z^{g}\right),
$$

then after rearranging, one finds

$$
\Theta_{a}\left(\alpha_{1}, \alpha_{2}\right)=e^{-\pi i t_{\alpha_{1}} \cdot \alpha_{2}} \cdot \sum_{n \in \alpha_{2}+Z^{z}} e^{2 \pi i\left[\frac{1}{2} t_{n} \cdot \tau \cdot n+t_{n} \cdot \alpha_{1}\right]} .
$$

The function so defined clearly extends to a locally constant function defined for all $\alpha_{1}, \alpha_{2} \in Q^{2}$ : it is the sought-for algebraic theta function defined in $\S 8$. Comparing this with the formula in Lemma $1, \S 8$, expressing $\Theta_{a}$ in terms of the finitely additive measure $\mu$ on $\boldsymbol{Q}_{2}^{z}$, we also get an analytic description for $\mu$ :

$$
\left\{\begin{array}{l}
\mu \text { is countably additive, } \\
\mu=\sum_{x \in D} e^{\pi i t_{x} \cdot \tau \cdot x} \cdot \delta_{x} \\
\delta_{x}=\text { delta measure at } x \\
D=\bigcup_{k} 2^{-k} Z^{g}
\end{array}\right.
$$

17 Inventiones math., Vol. 3 


\section{References}

[1] BAILY, jr., W.: On the theory of $\Theta$-functions, the moduli of abelian varieties, and the moduli of curves. Annals of Math. 75, 342-381 (1962).

[2] CARTIER, P.: Quantum mechanical commutation relations and theta functions. Proc. of Symposia in Pure Math., vol. 9. Am. Math. Soc. 1966.

[3] Grothendirck, A.: Séminaire de géométrie algébrique. Inst. des Hautes Études Sci. 1960/61 (Mimeographed).

[4] - Séminaire: Schémas en groupes. Inst. des Hautes Études Sci. 1963/64 (Mimeographed).

[5] -, et J. Dieudonne: Éléments de la géométrie algébrique. Publ. de l'Inst. des Hautes Études Sci., No. 4, 8, 11, etc.

[6] IgusA, J.-I.: On the graded ring of theta-constants. Am. J. Math. 86, 219-246 (1964); 88, 221 - 236 (1966).

[7] LANG, S.: Abelian Varieties. New York: Interscience 1958.

[8] Mackey, G.: On a theorem of Stone and von Neumann. Duke Math. J. 16, 313-330 (1949).

[9] Mumford, D.: Geometric invariant theory. Berlin-Heidelberg-New York: Springer 1965.

[10] - Curves on an algebraic surface. Annals of Math. Studies, No. 59 (1966).

[11] SiEgEL, C.: Moduln Abelscher Funktionen. Nachrichten der Akad., Göttingen 1964.

[12] WeIL, A.: Sur certaines groupes d'opérateurs unitaires. Acta Math. 111, 145-211 (1964).

[13] Iwahori, N., and H. Matsumoto: On some Bruhat decomposition and the structure of the Hecke rings of $p$-adic Chevalley Groups. Publ. de 1'Inst. des Hautes Etudes Sci., No. 25.

[14] Matsusaka, T.: Polarized varieties and fields of moduli. Am. J. of Math. 80, 45-82 (1958).

[15] GrothendiecK, A.: Fondements de la geometrie algebrique. Secretariat math., Paris (Mimeographed).

\section{Department of Mathematics Harvard University} Cambridge, Massachussetts

(Received February 20, 1967) 